\begin{tabular}{|c|l|}
\hline Title & Scaling limit of a model of quantum electrodynamics \\
\hline Author(s) & Hiroshima, F. \\
\hline Citation & Hokkaido University Preprint Series in Mathematics, 194, 1-53 \\
\hline Issue Date & 1993-04 \\
\hline DOI & 10.14943/83338 \\
\hline Doc URL & http:/hdl.handle.net/2115/68940 \\
\hline Type & bulletin (article) \\
\hline File Information & pre194.pdf \\
\hline
\end{tabular}

Instructions for use 
Scaling Limit of a Model of Quantum Electrodynamics

Fumio Hiroshima

Series $\sharp 194$. April 1993 


\section{HOKKAIDO UNIVERSITY \\ PREPRINT SERIES IN MATHEMATICS}

$\sharp 167$ : Y. Okabe, H. Mano and Y. Itoh; Random collision model for interacting populations of two species and its strong law of large numbers, 14 pages. 1992.

$\sharp 168: \quad$ A. Inoue, On the equations of stationary precesses with divergent diffusion coefficients, 25 pages. 1992.

\#169: T. Ozawa, Remarks on quadratic nonlinear Schrödinger equations, 19 pages. 1992.

$\sharp 170$ : T. Fukui, Y. Giga, Motion of a graph by nonsmooth weighted curvature, 11 pages. 1992.

$\sharp 171$ : J. Inoue, T. Nakazi, Finite dimensional solution sets of extremal problems in $\mathrm{H}^{1}, 10$ pages. 1992.

$\sharp 172$ : S. Izumiya, A characterization of complete integrability for partial differential equations of first order, 6 pages. 1992.

\#173: T. Suwa, Unfoldings of codimension one complex analytic foliation singularities, 49 pages. 1992.

$\sharp 174$ : T. Ozawa, Wave propagation in even dimensional spaces, 15 pages. 1992.

$\sharp 175$ : $\quad$ S. Izumiya, Systems of Clairaut type, 7 pages. 1992.

$\sharp 176$ : A. Hoshiga, The initial value problems for quasi-linear wave equations in two space dimensions with small data, 25 pages. 1992.

\#177: K. Sugano, On bicommutators of modules over H-separable extension rings III, 9 pages. 1993.

$\sharp 178$ : T. Nakazi, Toeplitz operators and weighted norm inequalities, 17 pages. 1993.

$\sharp 179$ : O. Ogurisu, Existence and structure of infinitely degenerate zero-energy ground states of a Wess-Zumino type model in supersymmetric quantum mechanics, 26 pages. 1993.

$\$ 180$ : O. Ogurisu, Ground state of a spin 1/2 charged particle in an even dimensional magnetic field, 9 pages. 1993.

$\sharp 181$ : K. Sugano, Note on H-separable Galois extension, 6 pages. 1993.

$\sharp 182$ : M. Yamada, Distance formulas of asymptotic Toeplitz and Hankel operators, 13 pages. 1993

$\sharp 183$ : G. Ishikawa, T. Ozawa, The genus of a connected compact real algebraic surface in the affine three space, 11 pages. 1993.

$\sharp 184$ : T. Hibi, Canonical modules and Cohen-Macaulay types of partially ordered sets, 6 pages. 1993.

$\sharp 185$ : Y. Giga, K. Yama-uchi, On a lower bound for the extinction time of surfaces moved by mean curvature, 16 pages. 1993.

\#186: Y. Kurokawa, On functional moduli for first order ordinary differential equations, 9 pages. 1993.

$\sharp 187$ : O. Ogurisu, Unitary equivalence between a spin 1/2 charged particle in a two-dimensional magnetic field and a spin $1 / 2$ neutral particle with an anomalous magnetic moment in a two-dimensional electric field, 4 pages. 1993.

$\sharp 188$ : A. Jensen, T. Ozawa, Existence and non-existence results for wave operators for perturbations of the laplacian, 30 pages. 1993.

$\sharp 189: \quad$ T. Nakazi, Multipliers of invariant subspaces in the bidisc, 12 pages. 1993.

$\sharp 190$ : S. Izumiya, G.T. Kossioris, Semi-local classification of geometric singularities for Hamilton-Jacobi equations, 24 pages. 1993.

\#191: N. Hayashi, T. Ozawa, Finite energy solutions of nonlinear Schrödinger equations of derivative type, 21 pages. 1993.

$\sharp 192$ : J. Seade, T. Suwa, A residue formula for the index of a holomorphic flow, 22 pages. 1993.

$\sharp 193$ : H. Kubo, Blow-up of solutions to semilinear wave equations with initial data of slow decay in low space dimensions, 8 pages. 1993. 


\title{
Scaling Limit of a Model of Quantum Electrodynamics
}

\author{
Fumio Hiroshima \\ Department of Mathematics, Hokkaido University \\ Sapporo 060, Japan
}

\begin{abstract}
This article gives a solution to an open problem in the paper: A.Arai; J.Math.Phys.31, $2653(1990)$. We present an abstract asymptotic theory of families of unitary operators $\{\mathcal{U}(\kappa)\}_{\kappa>0}$ and self-adjoint operators $\left\{H_{\kappa}\right\}_{\kappa>0}$ acting in the tensor product of two Hilbert spaces. We prove that $H_{\epsilon}^{r e n}(V, \kappa)$, which represents a scaled total Hamiltonian of a coupled system of a one electron atom and a quantized radiation field, with parameters $0 \leq \epsilon \leq 1, \kappa>0$, and the electron mass renormlized, is unitarily equivalent to an operator $\widetilde{H}_{\epsilon}^{r e n}(V, \kappa)$, which can be regarded as a decoupled Hamiltonian. Applying the abstract asymptotic theory and the unitary equivalence, we prove that the resolvent of $H_{\epsilon}^{r e n}(V, \kappa)$ strongly converges as $\kappa \rightarrow \infty$ to an operator which defines an effective potential of the electron. We compare the effective potential with that obtained in the paper mentioned above.
\end{abstract}




\section{INTRODUCTION}

This article gives a solution to an open problem in Ref.1.

We shall discuss a nonrelativistic limit of a model in quantum electrodynamics, which describes a nonrelativistic one electron atom coupled to a quantized radiation field. The nonrelativistic limit we study on the model is a scaling limit of the speed of light at the same time as the coupling constant of the model gets a scale transformation.

In Refs.2 and 3, the Pauli-Fierz model (stated below) is studied. It is well known that the Pauli-Fierz model is a realistic one in the sense that in a nonrelativistic region, it explains well some physical phenomena such as Lamb shift, although the explanations are usually done by formal perturbation calculation. In Ref.1, rigorous mathematical basis have been given to the Pauli-Fierz model by using a nonrelativistic limit. However the Pauli-Fierz model neglects the self-interaction term: $A^{2}$. In this paper we consider a model with the $A^{2}$-term.

Let $L^{2}\left(\mathbb{R}^{d}\right)$ and $\mathcal{F}^{E M}$ be the Hilbert spaces of state vectors of the electron and the quantized radiation field, respectively. The total Hamiltonian $H$ of the model is realized as a self-adjoint operator in the tensor product $L^{2}\left(\mathbb{R}^{d}\right) \otimes \mathcal{F}^{E M}$, and has parameters $c>0, e \in \mathbb{R} \backslash\{0\}, m>0$ denoting the speed of light, the elementary charge and the mass of the electron, respectively. Introducing scaled parameters $c(\kappa), e(\kappa), m(\kappa)$, with a scaling parameter $\kappa$, we define the scaled total Hamoiltonian $H(\kappa)$ by replacing the parameters $c, e, m$ by $c(\kappa), e(\kappa), m(\kappa)$, respectively.

The main purpose of this paper is to investigate an asymptotic behavior of the resolvent of $H(\kappa)$ as $\kappa . \rightarrow \infty$. Fot this purpose, we shall show the exsistence of a family of unitary operators $\{\hat{\mathcal{U}}(\kappa)\}_{\kappa>0}$ on $L^{2}\left(\mathbb{R}^{d}\right) \otimes \mathcal{F}^{E M}$ such that

$$
\widetilde{H}(\kappa) \equiv \widehat{\mathcal{U}}(\kappa)^{-1} H(\kappa) \widehat{\mathcal{U}}(\kappa)
$$

is a special version of the self-adjoint operator $H_{\kappa}$ discussed in Ref.1. In Ref.1, the effective operator $H_{\text {eff }}$ of $H_{\kappa}$ as $\kappa \rightarrow \infty$ is defined as a self-adjoint operator in $L^{2}\left(\mathbb{R}^{d}\right)$ by

$$
\lim _{\kappa \rightarrow \infty}\left(H_{\kappa}-z\right)^{-1}=\left(H_{e f f}-z\right)^{-1} \otimes P
$$

where $P$ is a projection operator on $\mathcal{F}^{E M}$. We show that $\hat{\mathcal{U}}(\kappa)$ strongly converges to a unitary 
operator $\widehat{\mathcal{U}}$ as $\kappa \rightarrow \infty$ and

$$
\lim _{\kappa \rightarrow \infty}(H(\kappa)-z)^{-1}=\hat{\mathcal{U}}\left(\left(\tilde{H}_{\text {eff }}-z\right)^{-1} \otimes P\right) \hat{\mathcal{U}}^{-1}
$$

where $\tilde{H}_{\text {eff }}$ is the effective operator of $\tilde{H}(\kappa)$ as $\kappa \rightarrow \infty$. We shall present an explicit form of $\tilde{H}_{\text {eff }}$, and compare it with an effective operator of Ref.1.

The outline of the present paper is as follows. In Section II, we review fundamental facts in the abstract Boson Fock space $\mathcal{F}(\mathcal{H})$ over a Hilbert space $\mathcal{H}$.

In Section III, we define a family of inhomogenous linear transformations of the canonical pair of field operartors $a^{\dagger}(f)$ and $a(f)$ in $\mathcal{F}(\mathcal{H})$ by the mappings:

$$
a^{\dagger}(f) \longrightarrow a^{\dagger}(\overline{S(\kappa)} f)+a(\overline{T(\kappa)} f)+(\overline{L(\kappa)}, f),
$$$$
a(f) \longrightarrow a^{\dagger}(T(\kappa) f)+a(S(\kappa) f)+(L(\kappa), f),
$$

where $0<\kappa<\infty,\{S(\kappa)\}$ and $\{T(\kappa)\}$ are two families of bounded linear operators on $\mathcal{H}$, and $\{L(\kappa)\}$ is a family of vectors in it. It is well known that under some conditions for $S(\kappa), T(\kappa)$ and $L(\kappa)$, the both sides of (1.1) are unitarily equivalent. We denote by $\{\mathcal{U}(\kappa)\}$ the family of the unitary operators on $\mathcal{F}(\mathcal{H})$ which implement the unitary equivalences. We shall show that under some conditions, $\mathcal{U}(\kappa)$ converges strongly to a unitary operator $\mathcal{U}$ as $\kappa \rightarrow \infty$.

In Section IV, we review an abstract theory of asymptotics of a family of self-adjoint operators $\left\{H_{\kappa}\right\}$ acting in the tensor product of two Hilbert spaces with a little modification of Ref.1 and define an effective operator $H_{\text {eff }}$ of $H_{\kappa}$ as $\kappa \rightarrow \infty$.

In Section V, we define our model. The Hamiltonian of the model without a potential for the electron is defined as a self-adjoint operator in $L^{2}\left(\mathbb{R}^{d}\right) \otimes \mathcal{F}^{E M}$ :

$$
H=H_{A} \otimes I+H_{I}^{(1)}+H_{I}^{(2)}+I \otimes H^{E M},
$$

which describes the interaction of one free electron and the quantized radiation field with the dipole approximation. In Ref.1, the model, without the term $H_{I}^{(2)}$, was considered, which is called the Pauli-Fierz model (Refs.2 and 3). In order to compare our model with the model of Ref.1, we shall introduce an operator in $L^{2}\left(\mathbb{R}^{d}\right) \otimes \mathcal{F}^{E M}$ by

$$
H_{\epsilon}=H_{A} \otimes I+H_{I}^{(1)}+\epsilon H_{I}^{(2)}+I \otimes H^{E M},
$$


where $0 \leq \epsilon \leq 1$. We show that $H_{\epsilon}$ is unitarily equivalent to an operator which can be regarded as a decoupled Hamiltonian $\widetilde{H}_{\epsilon}=H_{A}^{\prime} \otimes I+I \otimes H^{E M}$, and the unitary operator, which implments the unitary equivalence, is induced by the inhomogenous linear transformation on $\mathcal{F}^{E M}$ stated in Section III.

In Section VI, we shall discuss an asymptotic behavior of the total Hamiltonian with an electron mass renormalized, $H_{\epsilon}^{r e n}(V)=H_{\epsilon}^{r e n}+V$, where $V$ is the potential of the one electron atom and $H_{\epsilon}^{r e n}$ is the mass-renormalized coupled Hamiltonian. The operator $H_{\epsilon}^{r e n}(V)$ depends on parameters $c, e, m$. The scaled total Hamiltonian $H_{\epsilon}^{r e n}(V, \kappa)$ is defined by replacing the parameters $c, e, m$ by $c(\kappa), e(\kappa), m(\kappa)$, respectively, with $c(\kappa)=c \kappa, e(\kappa)=e \kappa^{-\frac{1}{2}}, m(\kappa)=m \kappa^{-2}$. The non-relativistic limit we study in this paper is taken in the sense of the scaling limit $\kappa \rightarrow \infty$. Since $|e(\kappa)|, m(\kappa) \rightarrow 0$ as $\kappa \rightarrow \infty$, the non-relativistic limit is a scaling limit of the speed of light at the same time as the magnitude of the elementary charge and the mass of the electron become 0 . Using the unitary equivalence relation of $H_{\epsilon}^{r e n}$ stated in Section $\mathrm{V}$, we show that $H_{\epsilon}^{r e n}(V, \kappa)$ is unitarily equivalent to a self-adjoint operator which is of the form of $H_{\kappa}$ discussed in Section IV:

$$
\tilde{H}_{\epsilon}^{r e n}(V, \kappa)=\hat{U}^{-1}(\kappa) H_{\epsilon}^{r e n}(\kappa) \hat{U}(\kappa)
$$

The unitary operator $\hat{\mathcal{U}}(\kappa)$ is just of the form of that in Section III. Applying the abstract theory in Sections III and IV, we find that the effective operator $\widetilde{H}_{\text {eff }}$ of $\widetilde{H}_{\epsilon}^{r e n}(V, \kappa)$ as $\kappa \rightarrow \infty$ satisfies

$$
s-\lim _{\kappa \rightarrow \infty}\left(H_{\epsilon}^{r e n}(\kappa)-z\right)^{-1}=\hat{U}\left(\left(\tilde{H}_{e f f}-z\right)^{-1} \otimes P\right) \hat{U}^{-1} .
$$

The effective operator $\tilde{H}_{\text {eff }}$ is of the form "Laplacian+Potential". Its potential is called an "effective potential". We show that the effective potential of $\tilde{H}_{\text {eff }}$ coincides with that of Refs. 1 and 3 .

In Appendix A, we prove Lemma 5.14. In Appendix B, we construct a generalized solution of the Heisenberg equation.

\section{FUNDAMENTAL FACTS IN AN ABSTRACT BOSON FOCK SPACE}

In this section we shall recall the definition of objects in an abstract Boson Fock space. Let $\mathcal{H}$ be a separable complex Hilbert space with norm $\|\cdot\|$ and inner product $(\cdot, *)$ (linear in $*$ ). Let $\mathrm{J}$ be a conjugation on $\mathcal{H}$, i.e., $\mathrm{J}$ is an antilinear isometry on $\mathcal{H}$ with $\mathrm{J}^{2}=I$, where $I$ is the identity on 
$\mathcal{H}$. For $f \in \mathcal{H}$ and $T \in \mathbf{B}(\mathcal{H})(\mathbf{B}(\mathcal{H})$ is the set of bounded linear operators on $\mathcal{H})$, we define $\bar{f} \in \mathcal{H}$ and $\bar{T} \in \mathbf{B}(\mathcal{H})$ by

$$
\bar{f}=\mathrm{J} f, \bar{T}=\mathbf{J} T \mathbf{J} .
$$

Let $\otimes_{s}^{n} \mathcal{H}$ be the $\mathrm{n}$-fold symmetric tensor product of $\mathcal{H}$ with convention $\otimes_{s}^{0} \mathcal{H}=\mathrm{C}$. The symmetric Boson Fock space $\mathcal{F}(\mathcal{H})$ over $\mathcal{H}$ is defined by

$$
\mathcal{F}(\mathcal{H})=\bigoplus_{n=0}^{\infty} \otimes_{s}^{n} \mathcal{H}
$$

Every element $\Phi \in \mathcal{F}(\mathcal{H})$ is a sequence $\Phi=\left\{\Phi^{(n)}\right\}_{n=0}^{\infty}$ of vectors $\Phi^{(n)} \in \otimes_{s}^{n} \mathcal{H}(n \geq 0)$ which satisfies

$$
\|\Phi\|^{2}=\sum_{n=0}^{\infty}\left\|\Phi^{(n)}\right\|^{2}<\infty
$$

We denote by $\mathcal{F}_{0}(\mathcal{H})$ the finite particle subspace

$$
\mathcal{F}_{0}(\mathcal{H})=\left\{\Phi=\left\{\Phi^{(n)}\right\}_{n=0}^{\infty} \in \mathcal{F}(\mathcal{H}) \mid \Phi^{(n)}=0 \text { for all but finitly many } n\right\}
$$

and by $a^{\dagger}(f),(f \in \mathcal{H})$ a creation operator in $\mathcal{F}(\mathcal{H})$ which is definded on $\mathcal{F}_{0}(\mathcal{H})$ and leaves it invariant satisfying the canonical commutation relations (CCR): for $f, g \in \mathcal{H}$,

$$
\begin{aligned}
& {[a(f), a(g)]=\left[a^{\dagger}(f), a^{\dagger}(g)\right]=0,} \\
& {\left[a(f), a^{\dagger}(g)\right]=(\bar{f}, g),}
\end{aligned}
$$

where $a(f)$ is the adjoint of $a^{\dagger}(\bar{f})\left\lceil\mathcal{F}_{0}(\mathcal{H})\right.$ (the annihilation operator in $\mathcal{F}(\mathcal{H})$ ) and

$$
[A, B] \equiv A B-B A \text {. }
$$

The operator $a^{\dagger}(\bar{f})\left\lceil\mathcal{F}_{0}(\mathcal{H})\right.$ is closable; we denote the closure by the same symbol. Let $\mathbf{C}(\mathcal{H})$ denote the set of densly defined closed linear operators in $\mathcal{H}$. For any $h \in \mathbf{C}(\mathcal{H})$, the second quantization $d \Gamma(h) \in \mathbf{C}(\mathcal{F}(\mathcal{H}))$ is defined as the infinite direct sum

$$
d \Gamma(h)=\bigoplus_{n=0}^{\infty} d \Gamma_{n}(h)
$$

with $d \Gamma_{0}(h)=0$ and

$$
d \Gamma_{n}(h)=\underbrace{h \otimes I \otimes \ldots \otimes I}_{\mathrm{n} \text {-times }}+\underbrace{I \otimes h \otimes \ldots \otimes I}_{\mathrm{n} \text {-times }}+\ldots+\underbrace{I \otimes I \otimes \ldots \otimes h}_{\mathrm{n} \text {-times }}(n \geq 1) .
$$


It is well known that if $h$ is a nonnegative self adjoint operator, then so is $d \Gamma(h)$, and $a^{\sharp}(f)$ : $D\left(d \Gamma(h)^{n+\frac{1}{2}}\right) \rightarrow D\left(d \Gamma(h)^{n}\right)\left(f \in D\left(h^{n+\frac{1}{2}}\right), n \geq 1\right)$, in particular for $f \in \bigcap_{n=0}^{\infty} D\left(h^{n}\right), a^{\sharp}(f)$ leaves $\bigcap_{n=0}^{\infty} D\left(d \Gamma(h)^{n}\right)$ invariant. The number operator $\mathrm{N}$ is given by the second quantization of $I$ :

$$
d \Gamma(I)=\mathbf{N}
$$

It follows that $\Phi \in D(\mathrm{~N})$ if and only if $\sum_{n=0}^{\infty} n^{2}\left\|\Phi^{(n)}\right\|^{2}<\infty$ and $(\mathrm{N} \Phi)^{(n)}=n \Phi^{(n)}$. The follwing facts are well-known:

$$
\begin{aligned}
& \left\|a^{\sharp}(f) \Phi\right\| \leq\|f\|\left\|(\mathrm{N}+1)^{\frac{1}{2}} \Phi\right\|, \Phi \in D\left(\mathrm{~N}^{\frac{1}{2}}\right), \\
& {\left[a^{\sharp}(f), \mathrm{N}\right] \Psi= \pm a^{\sharp}(f) \Psi, \Psi \in D\left(\mathrm{~N}^{\frac{3}{2}}\right),}
\end{aligned}
$$

where + in Eq. $(2.2)$ (resp. -) corresponds to $a(f)$ (resp. $a^{\dagger}(f)$ ). In addition to the subspace $\mathcal{F}_{0}(\mathcal{H})$, we introduce two useful dense subspaces $\mathcal{D}$ and $\mathcal{D}_{\infty}$ in $\mathcal{F}(\mathcal{H})$ as follows:

$$
\begin{aligned}
& \mathcal{D}=\mathcal{L}\left\{a^{\dagger}\left(f_{1}\right) a^{\dagger}\left(f_{2}\right) \ldots a^{\dagger}\left(f_{n}\right) \Omega, \Omega \mid f_{j} \in \mathcal{H}, j=1, \ldots, n, n \geq 1\right\} \\
& \mathcal{D}_{\infty}=\bigcap_{n=1}^{\infty} D\left(\mathbb{N}^{n}\right),
\end{aligned}
$$

where $\Omega$ denotes the Fock vacuum $\Omega=\{1,0,0 \ldots\}$ and $\mathcal{L}\{\ldots\}$ the set of finite linear combinations of $\{\ldots\}$. We recall that for all $f \in \mathcal{H}, a^{l}$ leaves $\mathcal{D}$ and $\mathcal{D}_{\infty}$ invariant. Further, using (2.1) and Eq.(2.2), one can easily see that if $f_{j, n} \rightarrow f_{j}$ as $n \rightarrow \infty, j=1, \ldots, N, N \geq 1$, then for $\Phi \in \mathcal{D}_{\infty}$,

$$
\lim _{n \rightarrow \infty}\left\|a^{\dagger}\left(f_{1, n}\right) \ldots a^{\dagger}\left(f_{N, n}\right) \Phi-a^{\dagger}\left(f_{1}\right) \ldots a^{\dagger}\left(f_{N}\right) \Phi\right\|=0 .
$$

It is well-known that for $f \in \mathcal{H}$ the operator

$$
\pi(f)=i\left(a^{\dagger}(f)-a(f)\right)
$$

is essentially self-adjoint operator on $\mathcal{F}_{0}(\mathcal{H})$.

Here we shall briefly collect some known results about operators quadratic in $a^{\sharp}$. The notations used below shall follow those of Ref.4. We denote the set of Hilbert-Schmidt operators on $\mathcal{H}$ by $I_{2}(\mathcal{H})$ with Hilbert-Schmidt norm $\|\cdot\|_{H S}$. For any $K \in I_{2}(\mathcal{H})$, there exist two orthonormal sets $\left\{\psi_{n}\right\}_{n=1}^{M},\left\{\phi_{n}\right\}_{n=1}^{M}$ in $\mathcal{H}$ and positve real numbers $\left\{\lambda_{n}\right\}_{n=1}^{M}$ (the non-zero eigenvalues of $|K|$ ) such that

$$
\sum_{n=1}^{M} \lambda_{n}^{2}<\infty
$$


and for all $f \in \mathcal{H}$

$$
K f=\sum_{n=1}^{M} \lambda_{n}\left(\psi_{n}, f\right) \phi_{n},
$$

where $M$ may be finite or infinite, but we shall consider the case $M=\infty$. For $K \in I_{2}(\mathcal{H})$, we put

$$
\begin{aligned}
& \left.<a^{\dagger}\left|K_{N}\right| a^{\dagger}\right\rangle \equiv \sum_{n=1}^{N} \lambda_{n} a^{\dagger}\left(\bar{\psi}_{n}\right) a^{\dagger}\left(\phi_{n}\right), \\
& \left.<a\left|K_{N}\right| a\right\rangle \equiv \sum_{n=1}^{N} \lambda_{n} a\left(\bar{\psi}_{n}\right) a\left(\phi_{n}\right) .
\end{aligned}
$$

Then it is well known that for all $\Phi \in \mathcal{D}$, the following limits exsist:

$$
\begin{aligned}
& <a^{\dagger}|K| a^{\dagger}>\Phi \equiv s-\lim _{N \rightarrow \infty}<a^{\dagger}\left|K_{N}\right| a^{\dagger}>\Phi \\
& <a|K| a>\Phi \equiv s-\lim _{N \rightarrow \infty}<a\left|K_{N}\right| a>\Phi
\end{aligned}
$$

Moreover, for all $K \in I_{2}(\mathcal{H})$,

$$
\left(<a^{\dagger}|K| a^{\dagger}>\Phi, \Psi\right)=\left(\Phi,<a\left|K^{*}\right| a>\Psi\right), \quad \Phi, \Psi \in \mathcal{D}
$$

It follows from Eq.(2.5) that $\left\langle a^{\|}|K| a^{\|}\right\rangle$is closable. We denote its closure by the same symbol. One can show that for vectors of the form $\Phi=a^{\dagger}\left(f_{1}\right) \ldots a^{\dagger}\left(f_{n}\right) \Omega\left(f_{j} \in \mathcal{K}, j=1, \ldots, n\right)$,

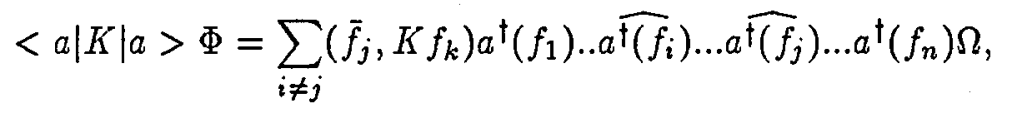

where ${ }^{-}$in Eq.(2.6) means that the operators with index $i, j$ are omitted.

By virtue of Eq.(2.6), we see that

$$
\begin{aligned}
& <a|K| a>^{m} \Phi=\sum_{j_{1} \neq . . \neq j_{2 m}}\left(\bar{f}_{j_{1}}, K f_{j_{2}}\right) \ldots\left(\bar{f}_{j_{2 m-1}}, K f_{j_{2 m}}\right),(n=2 m) \\
& <a|K| a>^{m} \Phi=0(2 m \geq n)
\end{aligned}
$$

For $S \in \mathrm{C}(\mathcal{H})$, we introduce the subspace in $\mathcal{F}(\mathcal{H})$

$$
\mathcal{D}_{S}=\mathcal{L}\left\{a^{\dagger}\left(f_{1}\right) a^{\dagger}\left(f_{2}\right) \ldots a^{\dagger}\left(f_{n}\right) \Omega, \Omega \mid f_{j} \in D(S), j=1,2, . ., n, n \geq 1\right\} .
$$

It can be easily seen that for $S \in \mathrm{C}(\mathcal{H})$ and $\Phi \in \mathcal{D}_{S}$, the strong limit

$$
:<a^{\dagger}|S| a>^{k}: \Phi \equiv s-\lim _{N \rightarrow \infty}:\left\{\sum_{n=1}^{N} a^{\dagger}\left(e_{n}\right) a\left(\overline{S^{*} e_{n}}\right)\right\}^{k}: \Phi
$$


exists independently of the choice of C.O.N.S. $\left\{e_{n}\right\}_{n=0}^{\infty}$ in $\mathcal{H}$ with $e_{n} \in D\left(S^{*}\right)(n \geq 1)$. Its action on the vectors of the form $\Phi=a^{\dagger}\left(f_{1}\right) \ldots a^{\dagger}\left(f_{n}\right) \Omega\left(f_{j} \in D(S), j=1, \ldots, n\right)$ is given by

$$
\begin{aligned}
& :<a^{\dagger}|S| a>^{k}: \Phi=k ! \sum_{n_{1}<\ldots n_{k}} a^{\dagger}\left(f_{1}\right) \ldots a^{\dagger}\left(S f_{n_{1}}\right) \ldots a^{\dagger}\left(S f_{n_{k}}\right) \ldots a^{\dagger}\left(f_{n}\right) \Omega,(k \leq n), \\
& :<a^{\dagger}|S| a>^{k}: \Phi=0,(k>n) .
\end{aligned}
$$

We shall next describe the commutation relations of $\left.\left\langle a^{\|}|K| a^{\sharp}\right\rangle,:<a^{\dagger}|S| a\right\rangle^{k}$ : and $a^{\sharp}$. For any operator $A$, we define $A^{T}=\overline{A^{*}}$.

It is well known that for $K_{j} \in I_{2}(\mathcal{H})(j=1, \ldots, n), f_{i} \in \mathcal{H}(i=1, \ldots, m)$ and $\Phi \in \mathcal{D}$,

$$
\begin{array}{r}
\Phi \in D\left(<a^{\dagger}\left|K_{1}\right| a^{\dagger}><a^{\dagger}\left|K_{2}\right| a^{\dagger}>\ldots<a^{\dagger}\left|K_{n}\right| a^{\dagger}>\right), \\
<a^{\dagger}\left|K_{1}\right| a^{\dagger}>\ldots<a^{\dagger}\left|K_{n}\right| a^{\dagger}>\Phi \in D\left(a^{\sharp}\left(f_{1}\right) \ldots a^{\sharp}\left(f_{m}\right)\right),
\end{array}
$$

and for $K \in I_{2}(\mathcal{H}), \Phi \in \mathcal{D}$

$$
\begin{aligned}
& {\left[a(f),<a^{\dagger}|K| a^{\dagger}>\right] \Phi=a^{\dagger}\left(K^{T}+K\right) \Phi,} \\
& {\left[a^{\dagger}(f),<a|K| a>\right] \Phi=-a\left(K^{T}+K\right) \Phi,} \\
& {[a(f),<a|K| a>] \Phi=\left[a^{\dagger}(f),<a^{\dagger}|K| a^{\dagger}>\right] \Phi=0 .}
\end{aligned}
$$

Similarly it can be easily seen that for $S \in \mathbf{C}(\mathcal{H}), f \in D(S), g \in D\left(S^{T}\right)$ and $\Phi \in \mathcal{D}_{S}$,

$$
\begin{aligned}
& {\left[:<a^{\dagger}|S| a>^{k}:, a^{\dagger}(f)\right] \Phi=k a^{\dagger}(S f):<a^{\dagger}|S| a>^{k-1}: \Phi} \\
& {\left[:<a^{\dagger}|S| a>^{k}:, a(g)\right] \Phi=-k a\left(S^{T} g\right):<a^{\dagger}|S| a>^{k-1}: \Phi .}
\end{aligned}
$$

We introduce two sets $\tilde{I}_{2}(\mathcal{H})$ and $\theta$ by

$$
\begin{aligned}
\tilde{I}_{2}(\mathcal{H}) & =\left\{K \in I_{2}(\mathcal{H}) \mid K=K^{T},\|K\|<1\right\} \\
\theta & =\left\{z \in \mathbb{C}|| z \mid<\|K\|^{-2}\right\}
\end{aligned}
$$

For $K \in I_{2}(\mathcal{H})$, we have

$$
\left\|\sum_{n=0}^{N} \frac{1}{n !}\left(-\frac{1}{2}<a^{\dagger}|K| a^{\dagger}>\right)^{n} \Omega\right\|^{2}=\sum_{n=0}^{N} a_{n},
$$

where $a_{n}=\left(2^{n} n !\right)^{-2}\left\|<a^{\dagger}|K| a^{\dagger}>^{n} \Omega\right\|^{2}$. 
Proposition 2.1 : Let $K \in \bar{I}_{2}(\mathcal{H})$. Then the following (1) - (4) hold:

(1) For all $z \in \theta$ and $k \geq 0$, the limit $\lim _{N \rightarrow \infty} \sum_{n=0}^{N} n^{k} a_{n} z^{n}$ exists.

(2) $\sum_{n=0}^{\infty} a_{n} z^{n}=\operatorname{det}\left(I-z K^{*} K\right)^{-\frac{1}{2}}, z \in \theta$.

(3) $\sum_{n=0}^{\infty} n^{2 k} a_{n}=\left\|\mathrm{N}^{k} \Omega(K)\right\|^{2}, k \geq 0$.

(4) For all $\Phi \in \mathcal{D}$, the strong limit

$$
\exp \left(-\frac{1}{2}<a^{\dagger}|K| a^{\dagger}>\right) \Phi \equiv s-\lim _{N \rightarrow \infty} \sum_{n=0}^{N} \frac{1}{n !}\left(-\frac{1}{2}<a^{\dagger}|K| a^{\dagger}>\right)^{n} \Phi,
$$

exists and belongs to $\mathcal{D}_{\infty}$.

Proof: See Refs.5,6 and 7.

For $L \in I_{2}(\mathcal{H}), S \in \mathrm{C}(\mathcal{H}), \Psi \in \mathcal{D}$ and $\Phi \in \mathcal{D}_{S}$, we define

$$
\begin{aligned}
& : \exp \left(-\frac{1}{2}<a^{\dagger}|S| a>\right): \Phi \equiv s-\lim _{N \rightarrow \infty} \sum_{n=0}^{N} \frac{1}{n !}:\left(-\frac{1}{2}<a^{\dagger}|S| a>\right)^{n}: \Phi, \\
& \exp \left(-\frac{1}{2}<a|L| a>\right) \Phi \equiv s-\lim _{N \rightarrow \infty} \sum_{n=0}^{N} \frac{1}{n !}\left(-\frac{1}{2}<a|L| a>\right)^{n} \Phi .
\end{aligned}
$$

In the summation of the definitions (2.9) and (2.10), the number of non-zero terms is finite. Hence the definitions (2.9) and (2.10) are well defined and the both belong to $\mathcal{D}$. By virtue of of Proposition 2.1 , we can define for $K \in \tilde{I}_{2}(\mathcal{H})$, a vector $\Omega(K) \in \mathcal{D}_{\infty}$ by

$$
\Omega(K)=\mathcal{N} \exp \left(-\frac{1}{2}<a^{\dagger}|K| a^{\dagger}>\right) \Omega
$$

where $\mathcal{N}$ is a normalized constant so that $\|\Omega(K)\|=1$. By Proposition $2.1(2), \mathcal{N}$ is explicitly given by

$$
\mathcal{N}=\operatorname{det}\left(I-K^{*} K^{-\frac{1}{4}}=\prod_{j=1}^{\infty}\left(1-\lambda_{j}^{2}\right)^{\frac{1}{4}} .\right.
$$

Lemma 2.2 : Let $K \in \tilde{I}_{2}(\mathcal{H}), L \in I_{2}(\mathcal{H}), S \in \mathrm{C}(\mathcal{H})$, and put

$$
\begin{aligned}
& U_{1}(K)=\exp \left(-\frac{1}{2}<a^{\dagger}\left|K^{*}\right| a^{\dagger}>\right), \\
& U_{2}(S)=\exp \left(-\frac{1}{2}<a^{\dagger}|S| a>\right): \\
& U_{3}(L)=\exp \left(-\frac{1}{2}<a|L| a>\right) .
\end{aligned}
$$


For $\Phi \in \mathcal{D}$ and $\Psi \in \mathcal{D}_{S}$, the following commutation relations hold.

(1) $\left[U_{1}(K), a(f)\right] \Phi=a^{\dagger}(K f) U_{1}(K) \Phi, f \subset \mathcal{H}$.

(2) $\left[U_{1}(K), a^{\dagger}(f)\right] \Phi=0, f \in \mathcal{H}$.

(3) $\left[U_{2}(S), a(f)\right] \Psi=a\left(\frac{1}{2} S^{T} f\right) U_{2}(S) \Psi, f \in D\left(S^{T}\right)$.

(4) $\left[U_{2}(S), a^{\dagger}(f)\right] \Psi=a^{\dagger}\left(-\frac{1}{2} S f\right) U_{2}(S) \Psi, f \in D(S)$.

(5) $\left[U_{3}(L), a(f)\right] \Phi=0, f \in \mathcal{H}$.

(6) $\left[U_{3}(L), a^{\dagger}(f)\right] \Phi=-\frac{1}{2} a\left(\left(L^{T}+L\right) f\right) U_{3}(L) \Phi . f \in \mathcal{H}$.

Proof: It is straightforwardly seen that on $\mathcal{D}$

$$
\begin{aligned}
-\quad & \left.\left.\sum_{n=0}^{N} \frac{1}{n !}\left(-\frac{1}{2}<a^{\dagger}|K| a^{\dagger}\right\rangle\right)^{n} a(f)-\sum_{n=0}^{N-1} \frac{1}{n !}\left(-\frac{1}{2}<a^{\dagger}|K| a^{\dagger}\right\rangle\right)^{n} a^{\dagger}(K f) \\
& \left.=a(f) \sum_{n=0}^{N} \frac{1}{n !}\left(-\frac{1}{2}<a^{\dagger}|K| a^{\dagger}\right\rangle\right)^{n} .
\end{aligned}
$$

The strong limit of the r.h.s. of (2.11) exists. Then, from the closedness of $a(f),(1)$ follows. The proofs of (2)-(6) are similar to that of (1), and the details are left to the reader.

Lemma 2.2 immediately gives the following corollary.

Corollary 2.3 : Let $K_{1} \in I_{2}(\mathcal{H}), K_{2}$ and $K_{2}^{-1}$ be in $\mathbf{B}(\mathcal{H})$ and $K_{1} K_{2}^{-1} \in \tilde{I}_{2}(\mathcal{H})$. Then

$$
\left\{a^{\dagger}\left(K_{1} f\right)+a\left(K_{2} f\right)\right\} \Omega\left(K_{1} K_{2}^{-1}\right)=0, \quad f \in \mathcal{H}
$$

Proof: By Lemma $2.2(1)$, we can see that

$$
a\left(K_{2} f\right) U_{1}\left(K_{1} K_{2}^{-1}\right) \Omega=-a^{\dagger}\left(K_{1} f\right) U_{1}\left(K_{1} K_{2}^{-1}\right) \Omega .
$$

Since $U_{1}\left(K_{1} K_{2}^{-1}\right) \Omega=\Omega\left(K_{1} K_{2}^{-1}\right)$, the desired result follows.

Proposition 2.4 : Let $K \in \tilde{I}_{2}(\mathcal{H}), S \in \mathrm{C}(\mathcal{H}), S^{T}\left(K+K^{T}\right)^{*} \in I_{2}(\mathcal{H})$. Then,

$$
\begin{aligned}
& \text { (1) }<a^{\dagger}|K| a^{\dagger}>^{k} \Omega \in D\left(d \Gamma(S)^{*}\right), \\
& \text { (2) } d \Gamma(S)^{*}<a^{\dagger}|K| a^{\dagger}>^{k} \Omega=k<a^{\dagger}\left|\left(K+K^{T}\right) \bar{S}\right| a^{\dagger}><a^{\dagger}\left|K^{\dagger}\right| a^{\dagger}>^{k-1} \Omega, \\
& \text { (3) } \Omega(K) \in D\left(d \Gamma^{*}(S)\right) .
\end{aligned}
$$

Proof: See Ref.8. 


\section{CANONICAL TRANSFORMS IN AN ABSTRACT BOSON FOCK SPACE}

In this section we shall investigate a family of inhomogenous linear transformations of the canonical pair of field operators $a^{\dagger}(f)$ and $a(f)$.

\subsection{Fundamental facts}

An inhomogenous linear transformation of the canonical pair of field operators $a^{\dagger}(f)$ and $a(f)$ is induced by $L \in \mathcal{H}$ and the sympletic group $\Sigma$ over $\mathcal{H}$. For bounded linear operators $T$ and $S$ on $\mathcal{H}$, we say that the matrix

$$
\Xi=\left(\begin{array}{cc}
S & T \\
\bar{T} & \bar{S}
\end{array}\right)
$$

is in the symmpletic group $\Sigma$ if and only if following relation holds on $\mathcal{H} \oplus \mathcal{H}$ :

$$
\Xi^{*} \mathrm{~J} \Xi=\Xi \Xi^{*}=\mathrm{J}
$$

where

$$
\mathrm{J}=\left(\begin{array}{cc}
I & 0 \\
0 & -I
\end{array}\right) \text { and } \Xi^{*}=\left(\begin{array}{cc}
S^{*} & \overline{T^{*}} \\
T^{*} & \overline{S^{*}}
\end{array}\right) .
$$

Throughtout this subsection, we fix $\Xi \in \Sigma$ with matrix elements $S$ and $T$ being in $\mathbf{B}(\mathcal{H})$. Let $L \in \mathcal{H}$. The inhomogenous linear transformation induced by $\Xi$ and $L$ is then defined as the mapping:

$$
\begin{aligned}
& a^{\dagger}(f) \longrightarrow a^{\dagger}(\bar{S} f)+a(\bar{T} f)+(\bar{L}, f) \equiv B^{\dagger}(f), \\
& a(f) \longrightarrow a^{\dagger}(T f)+a(S f)+(L, f) \equiv B(f) .
\end{aligned}
$$

For simplicity, we set

$$
\begin{aligned}
& b^{\dagger}(f)=a^{\dagger}(\bar{S} f)+a(\bar{T} f), \\
& b(f)=a^{\dagger}(T f)+a(S f) .
\end{aligned}
$$

In what follows, the notations $b^{\sharp}$ and $B^{!}$denotes $b^{\dagger}$ or $b$ and $B^{\dagger}$ or $B$, respectively.

Equation (3.1) implies the following relations:

$$
\begin{aligned}
& S^{*} S-T^{*} T=I, \\
& \overline{S^{*} T}-\overline{T^{*}} S=0,
\end{aligned}
$$




$$
\begin{aligned}
& S S^{*}-\overline{T T^{*}}=I, \\
& T S^{*}-\overline{S T^{*}}=0 .
\end{aligned}
$$

One can see that $b^{\sharp}(f)$ is defined on $\mathcal{F}_{0}(\mathcal{H})$ and leaves it invariant, and satisfies

$$
\begin{aligned}
& (b(f) \Psi, \Phi)=\left(\Psi, b^{\dagger}(\bar{f}) \Phi\right), \\
& {[b(f), b(g)] \Phi=\left[b^{\dagger}(f), b^{\dagger}(g)\right] \Phi=0} \\
& {\left[b(f), b^{\dagger}(g)\right] \Phi=(\bar{f}, g) \Phi,}
\end{aligned}
$$

for $\Phi, \Psi \in \mathcal{F}_{0}(\mathcal{H})$ and $f, g \in \mathcal{H}$. Furthermore by Eqs.(3.4) and (3.5), the operator $a^{\sharp}(f)$ can be represented as follows:

$$
\begin{aligned}
& a(f)=-B^{\dagger}\left(T^{T} f\right)+B\left(S^{*} f\right)-(S \bar{L}-\bar{T} L, f) \\
& a^{\dagger}(f)=B^{\dagger}\left(S^{T} f\right)-B\left(T^{*} f\right)-(\bar{S} L-T \bar{L}, f) .
\end{aligned}
$$

The adjoint-relation Eq.(3.6) implies that $b^{\sharp}(f)$ is closable; we denote its closure by the same symbol. In addition to the above condition $\Xi \in \Sigma$, we further suppose that

$$
T \in I_{2}(\mathcal{H})
$$

It is well known that under the condition $\Xi \in \Sigma,(3.9)$ is a necessary and sufficient condition for that there exists a unitary operator $\mathcal{U}$ (up to constant multiples with absolute value 1 ) on $\mathcal{F}$ such that

$$
\mathcal{U}^{-1} B^{\sharp}(f) \mathcal{U}=a^{\sharp}(f)
$$

This section is devoted to representing the unitary operator $\mathcal{U}$ explicitly. As we shall see below, the unitary operator $\mathcal{U}$ consists of two different unitary operators $U$ and $S$ on $\mathcal{F}(\mathcal{H})$, which are called the Intertwining operator and the Displacement operator, respectively (Ref.9):

$$
\mathcal{U}=\mathrm{SU}
$$

Before proceeding to the construction of these operators we present some technical lemmas.

Lemma 3.1 : The operator $S$ is bijective with

$$
\text { (1) }\left\|T S^{-1}\right\|<1, \quad(2)\left\|S^{-1}\right\| \leq\|S\| \text {. }
$$


Proof: It follows from Eqs.(3.2) and (3.4) that $S^{*} S$ and $S S^{*}$ are bounded and strictly positive selfadjoint operators on $\mathcal{H}$. This implies that $S$ is bijective. Further, from Eq.(3.2) and a bijectivity of $S^{-1}$, (1) follows. From the inequality $\left\|S^{-1}\right\|=\left\|\left(I+T^{*} T\right)^{-1} S^{*}\right\| \leq\left\|S^{*}\right\|=\|S\|$, (2) follows.

By Lemma 3.1 we can define three operators $K_{1}, K_{2}, K_{3}$ on $\mathcal{H}$ as follows:

$$
\begin{aligned}
& K_{1}=T S^{-1}, \\
& K_{2}=\bar{S}-T S^{-1} \bar{T}-I, \\
& K_{3}=S^{-1} \bar{T} .
\end{aligned}
$$

By Eqs.(3.2) and (3.3), it can be easily seen that

$$
K_{1}^{T}=K_{1}, K_{3}^{T}=K_{3}, K_{2}^{T}=S^{-1}-I
$$

Lemmas 3.1 and Eq.(3.10) imply that $K_{1} \in \tilde{I}_{2}(\mathcal{H})$, and from the definition of $K_{2}, K_{3}$ it follows that $K_{2} \in \mathrm{B}(\mathcal{H})$ and $K_{3} \in I_{2}(\mathcal{H})$. Thus we can define operators $U_{1}, U_{2}$ and $U_{3}$ in $\mathcal{F}(\mathcal{H})$ as follows:

$$
\begin{aligned}
& U_{1}=\exp \left(-\frac{1}{2}<a^{\dagger}\left|K_{1}\right| a^{\dagger}>\right), \\
& U_{2}=: \exp \left(-\frac{1}{2}<a^{\dagger}\left|2\left(-K_{2}\right)\right| a>\right): \\
& U_{3}=\exp \left(-\frac{1}{2}<a\left|\left(-K_{3}\right)\right| a>\right) .
\end{aligned}
$$

Since $U_{2}, U_{3}: \mathcal{D} \rightarrow \mathcal{D}$ and $U_{1}: \mathcal{D} \rightarrow \mathcal{D}_{\infty}$, the operator

$$
\mathrm{U}=\mathcal{N} U_{1} U_{2} U_{3}
$$

can be defined on $\mathcal{D}$, which maps from $\mathcal{D}$ to $\mathcal{D}_{\infty}$. The normalized factor $\mathcal{N}$ is explicitly given by

$$
\mathcal{N}=\operatorname{det}\left(I-K_{1}^{*} K_{1}\right)^{\frac{1}{4}}
$$

Lemma 3.2 : The operator $U$ can be uniquely extended to a unitary operator on $\mathcal{F}(\mathcal{H})$ with

$$
\mathrm{U}^{-1} b^{\sharp}(f) \mathrm{U}=a^{\sharp}(f), f \in \mathcal{H} .
$$

Proof: We begin with showing that Eq.(3.11) holds on $\mathcal{D}$. By the commutation relations of $U_{j}$ $(j=1,2,3$,$) and a^{\sharp}(f)$ (Lemma 2.2$)$, we have the following equations on $\mathcal{D}$,

$$
\begin{aligned}
U_{1} U_{2} U_{3} a^{\dagger}(f) & =U_{1} U_{2} a^{\dagger}(f) U_{3}+U_{1} U_{2} a\left(K_{3} f\right) U_{3} \\
& =a^{\dagger}\left(\left(I+K_{2}\right) f\right) U_{1} U_{2} U_{3}+U_{1} U_{2} a\left(K_{3} f\right) U_{3} .
\end{aligned}
$$


Using the fact that $a^{\dagger}\left(\left(I+K_{2}\right) f\right)=b^{\dagger}(f)+a(-\bar{T} f)+a^{\dagger}\left(-T S^{-1} \bar{T} f\right)$, we have

$$
\begin{aligned}
a^{\dagger}\left(\left(I+K_{2}\right) f\right) U_{1} U_{2} U_{3}= & b^{\dagger}(f) U_{1} U_{2} U_{3}+U_{1} a^{\dagger}\left(-T S^{-1} \bar{T} f\right) U_{2} U_{3} \\
& +U_{1}\left\{a^{\dagger}\left(K_{1} \bar{T} f\right)+a(-\bar{T} f)\right\} U_{2} U_{3} .
\end{aligned}
$$

Hence it follows

$$
E q .(3.13)=b^{\dagger}(f) U_{1} U_{2} U_{3}+U_{1} U_{2}\left\{a(-\bar{T} f)+a\left(-K_{2}^{T} \bar{T} f\right)\right\} U_{3} .
$$

Combining Eqs.(3.12) with (3.14), we obtain

$$
U_{1} U_{2} U_{3} a^{\dagger}(f)=b^{\dagger}(f) U_{1} U_{2} U_{3}+U_{1} U_{2} a\left(-\bar{T} f-K_{2}^{T} \bar{T} f+K_{3} f\right) U_{3} .
$$

Since Eq.(3.10) gives that $-\bar{T}-K_{2}^{T} \bar{T}+K_{3}=0$, we get for all $\Phi \in \mathcal{D}$ and $f \in \mathcal{H}$,

$$
\mathrm{U} a^{\dagger}(f) \Phi=b^{\dagger}(f) \mathrm{U} \Phi .
$$

Then Eq.(3.11) holds on $\mathcal{D}$. Next we put $U \Omega=\widetilde{\Omega}\left(\mathrm{U} \Omega=\mathcal{N} U_{1} \Omega\right)$ and set

$$
\mathcal{G}=\mathcal{L}\left\{b^{\dagger}\left(f_{1}\right) \ldots b^{\dagger}\left(f_{n}\right) \widetilde{\Omega}, \tilde{\Omega} \mid f_{j} \in \mathcal{H}, j=1, \ldots, n, n \geq 1\right\} .
$$

Since we have already seen that $a^{\dagger}$ can be represented by $b^{\sharp}$ and for all Corollary 2.3 gives $f \in \mathcal{H}$, $b(f) \tilde{\Omega}=0$, the subset $\mathcal{G}$ is irreducible with respect to $a^{\dagger}$ in the sense of Berezin. Then we can get $\overline{\mathcal{G}}=\mathcal{F}(\mathcal{H})$. Furthermore by Lemma 3.2 and the commutation relation of $b^{1}$, we see that $\mathrm{U}$ satisfy that

$$
\left\|\mathrm{U} a^{\dagger}\left(f_{1}\right) \ldots a^{\dagger}\left(f_{n}\right) \Omega\right\|=\left\|b^{\dagger}\left(f_{1}\right) \ldots b^{\dagger}\left(f_{n}\right) \tilde{\Omega}\right\|=\left\|a^{\dagger}\left(f_{1}\right) \ldots a^{\dagger}\left(f_{n}\right) \Omega\right\|
$$

Hence we conclude that $U$ maps $\mathcal{D}$ onto $\mathcal{G}$ and is isometry. Hence it can be uniquely extended to a unitary operator on $\mathcal{F}(\mathcal{H})$. We denote the unique extension by the same symbol. Since $b^{\dagger}(f)$ is closed, so is $\mathrm{U}^{-1} b^{\dagger}(f) \mathrm{U}$. Because $\mathcal{D}$ is a core of $a^{\sharp}$, taking the closure of both sides of (3.15) on $\mathcal{D}$ gives the desired result. The proof for $b(f)$ is similar to that of $b^{\dagger}(f)$. Thus we get Lemma 3.2.

We define the operator $\pi(L)$ in $\mathcal{F}(\mathcal{H})$ by

$$
\begin{aligned}
\pi(L) & =i\left\{b(L)-b^{\dagger}(\bar{L})\right\} \\
& =i\left\{a^{\dagger}(R)-a(\bar{R})\right\},
\end{aligned}
$$

where $R=T L-\overline{S L}$. Thus $\pi(L)$ is essentialy self-adjoint on $\mathcal{F}_{0}(\mathcal{H})$. We denote its closure by the same symbol. 
Lemma 3.3 : Let $\mathbf{S}=\exp (-i \pi(L))$. Then we have

$$
\mathbf{S} b^{\sharp}(f) \mathbf{S}^{-1}=B^{\sharp}(f) .
$$

Proof: For $\Phi, \Psi \in \mathcal{F}_{0}(\mathcal{H})$, since $\mathcal{F}_{0}(\mathcal{H})$ is the total set of analytic vectors of $\pi(L)$, we have

$$
\begin{aligned}
\left(\mathbf{S}^{-1} \Phi, b(f) \mathbf{S}^{-1} \Psi\right)= & \sum_{n, m} \frac{(-i)^{n-1}}{(n-1) !} \frac{i^{m}}{m !}\left((\bar{L}, \bar{f}) \pi(L)^{n-1} \Phi, \pi(L)^{m} \Psi\right) \\
& +\sum_{n, m} \frac{(-i)^{n}}{n !} \frac{i^{m}}{m !}\left(\pi(L)^{n} b^{\dagger}(\bar{f}) \Phi, \pi(L)^{m} \Psi\right) \\
= & (\Phi, B(f) \Psi) .
\end{aligned}
$$

Thus Eq.(3.16) holds on $\mathcal{F}_{0}(\mathcal{H})$. Since $b(f)$ is closed and $\mathcal{F}_{0}(\mathcal{H})$ is a core of $b(f)$, we get Eq.(3.16).

The proof for $b^{\dagger}(f)$ is similar to that of $b(f)$.

Theorem 3.4: We put $\mathcal{U}=\mathrm{SU}$. Then we have

$$
\mathcal{U}^{-1} B^{\sharp}(f) \mathcal{U}=a^{\sharp}(f), f \in \mathcal{H}
$$

Proof: This follows from combining Lemmas 3.2 and 3.3.

\subsection{The strong convergence of the Intertwining operator and the Displacement operator}

In this subsection we shall present an abstract asymptotic theory of a family of unitary operators $\{\mathcal{U}(\kappa)\}_{0<\kappa<\infty}$, which is induced by the cannonical pair of field operators $a^{\sharp}$, on $\mathcal{F}(\mathcal{H})$.

We shall assume that families of bounded operators $\{S(\kappa)\}_{\kappa>0}$, Hilbert-Schmidt operators $\{T(\kappa)\}_{\kappa>0}$ on $\mathcal{F}(\mathcal{H})$ and vectors $\{L(\kappa)\}_{\kappa>0}$ in $\mathcal{H}$ satisfy the following conditions;

(A.1): For all $0<\kappa<\infty$,

$$
\left(\frac{S(\kappa)}{T(\kappa)} \frac{T(\kappa)}{S(\kappa)}\right) \in \Sigma .
$$

(A.2): There exist a Hilbert-Schmidt opertator $T$ and a bounded linear operator $S$ on $\mathcal{F}(\mathcal{H})$ such that

$$
\begin{aligned}
& \lim _{\kappa \rightarrow \infty} T(\kappa)=T, \text { in strong topology, } \\
& \lim _{\kappa \rightarrow \infty} S(\kappa)=S, \text { in operator norm. }
\end{aligned}
$$


(A.3): The trace class operator $T^{*}(\kappa) T(\kappa)$ holds

$$
\lim _{\kappa \rightarrow \infty} T^{*}(\kappa) T(\kappa)=T^{*} T, \text { in trace norm. }
$$

(A.4): There exists a vector $L$ in $\mathcal{H}$ such that

$$
s-\lim _{\kappa \rightarrow \infty} L(\kappa)=L
$$

From conditions $(A .1)$ and $(A .2)$, it follows

$$
\left(\begin{array}{cc}
S & T \\
\bar{T} & \bar{S}
\end{array}\right) \in \Sigma .
$$

We define

$$
\begin{aligned}
& B_{\kappa}(f)=a^{\dagger}(T(\kappa) f)+a(S(\kappa) f)+(L(\kappa), f), 0<\kappa<\infty \\
& B_{\kappa}^{\dagger}(f)=a^{\dagger}(\overline{S(\kappa)} f)+(\overline{L(\kappa)}, f), 0<\kappa<\infty \\
& B(f)=a^{\dagger}(T f)+a(S f)+(L, f) \\
& B^{\dagger}(f)=a^{\dagger}(\bar{S} f)+a(\bar{T} f)+(\bar{L}, f)
\end{aligned}
$$

For simplicity, we put

$$
\begin{aligned}
& b_{\kappa}(f)=a^{\dagger}(T(\kappa) f)+a(S(\kappa) f), 0<\kappa<\infty, \\
& b_{\kappa}^{\dagger}(f)=a^{\dagger}(\overline{S(\kappa)} f)+a(\overline{T(\kappa)} f), 0<\kappa<\infty, \\
& b(f)=a^{\dagger}(T f)+a(S f), \\
& b^{\dagger}(f)=a^{\dagger}(\bar{S} f)+a(\bar{T} f) .
\end{aligned}
$$

As was mentioned in subsection 3.1, the mapping $a^{\sharp} \rightarrow B_{\kappa}^{\sharp}$ induces families of the Intertwining and the Displacement operators $\{\mathrm{U}(\kappa)\}_{0<\kappa<\infty}$ and $\{\mathrm{S}(\kappa)\}_{0<\kappa<\infty}$, and the mapping $a^{\sharp} \rightarrow B^{\sharp}, \mathrm{U}$ and $\mathrm{S}$, respectively. Putting $\mathcal{U}(\kappa)=\mathrm{S}(\kappa) \mathrm{U}(\kappa)$ and $\mathcal{U}=\mathrm{SU}$, we have

$$
\begin{aligned}
& a^{\sharp}(f)=\mathcal{U}(\kappa)^{-1} B_{\kappa}^{\sharp}(f) \mathcal{U}(\kappa), 0<\kappa<\infty, \\
& a^{\sharp}(f)=\mathcal{U}^{-1} B^{\sharp}(f) \mathcal{U} .
\end{aligned}
$$

Much of this subsection shall be devoted to the investigation of asymptotic behavior of the family $\{\mathcal{U}(\kappa)\}_{0<\kappa<\infty}$ as $\kappa \rightarrow \infty$. Since by Lemma3.1, the bijectivities of the bounded linear operators 
$S(\kappa)$ and $S$ are guaranteed, the operators $T(\kappa) S^{-1}(\kappa), 0<\kappa<\infty$, and $T S^{-1}$ are well defined and Hilbert-Schmidt operators, respectively. In the precisely same fashion as in Section II, we define operators $K_{j}(\kappa)$ and $K_{j},(j=1,2,3)$ on $\mathcal{F}(\mathcal{H})$ as follows:

$$
\begin{array}{ll}
K_{1}(\kappa)=T(\kappa) S^{-1}(\kappa), & K_{1}=T S^{-1}, \\
K_{2}(\kappa)=\overline{S(\kappa)}-T(\kappa) S^{-1}(\kappa) \bar{T}(\kappa)-I, & K_{2}=\bar{S}-T S^{-1} \bar{T}-I, \\
K_{3}(\kappa)=S^{-1}(\kappa) \overline{T(\kappa)}, & K_{3}=S^{-1} \bar{T} .
\end{array}
$$

We denote a trace norm by $\|\cdot\|_{T R}$.

Lemma 3.5: The limit of the trace class operator $K_{1}^{*}(\kappa) K(\kappa)$ as $\kappa \rightarrow \infty$ in the trace norm exists with

$$
\lim _{\kappa \rightarrow \infty}\left\|K_{1}^{*}(\kappa) K_{1}(\kappa)-K_{1}^{*} K_{1}\right\|_{T R}=0 .
$$

Proof: This follows directly from Lemma 3.1 (2), conditions $(A .2),(A .3)$ and two inequalities

$$
\begin{aligned}
\left\|K_{1}^{*}(\kappa) K_{1}(\kappa)-K_{1}^{*} K_{1}\right\|_{T R} \leq & \left(\left\|S^{-1}(\kappa)\right\|\|T(\kappa)\|_{H S}^{2}+\left\|S^{-1}\right\|\|T\|_{H S}^{2}\right)\left\|S^{-1}(\kappa)-S^{-1}\right\| \\
& +\left\|S^{-1}\right\|\left\|S^{-1}(\kappa)\right\|\left\|T^{*}(\kappa) T(\kappa)-T^{*} T\right\|_{T R}, \\
\left\|S^{-1}(\kappa)-S^{-1}\right\| \leq & \left\|S^{-1}(\kappa)\right\|\left\|S^{-1}\right\|\|S(\kappa)-S\| .
\end{aligned}
$$

We define two sets in $\mathbb{C}, \vartheta_{\kappa}$ and $\vartheta_{\infty}$, by

$$
\begin{aligned}
& \vartheta_{\kappa}=\left\{z \in \mathbb{C}|| z \mid<\left\|K_{1}(\kappa)\right\|^{-2}\right\}, 0<\kappa<\infty, \\
& \vartheta_{\infty}=\left\{z \in \mathbb{C}|| z \mid<\left\|K_{1}\right\|^{-2}\right\},
\end{aligned}
$$

and put the intersection of these sets by

$$
\vartheta=\bigcap_{0<\kappa<\infty} \vartheta_{\kappa} \bigcap \vartheta_{\infty}
$$

Let $\dot{\vartheta}$ be the interior domain of the closed disk $\bar{\vartheta}$. From the fact that $\lim \kappa \rightarrow \infty\left\|K_{1}(\kappa)\right\|=\left\|K_{1}\right\|$, and $\left\|K_{1}(\kappa)\right\|$ and $\left\|K_{1}\right\|$ are less than 1 , it follows that 1 is contained in $\dot{\vartheta}$. On account of Proposition 2.1, for $z \in \dot{\vartheta}$, we have

$$
\begin{aligned}
& \sum_{n=0}^{\infty} b_{n}(\kappa) z^{n}=\operatorname{det}\left(I-z K_{1}^{*}(\kappa) K_{1}(\kappa)\right)^{-\frac{1}{2}}, 0<\kappa<\infty \\
& \sum_{n=0}^{\infty} b_{n} z^{n}=\operatorname{det}\left(I-z K_{1}^{*} K_{1}\right)^{-\frac{1}{2}}
\end{aligned}
$$


where $b_{n}(\kappa)=\frac{1}{2^{n} n !}\left\|<a^{\dagger}\left|K_{1}(\kappa)\right| a^{\dagger}>^{n} \Omega\right\|^{2}$ and $b_{n}=\frac{1}{2^{n} n !}\left\|<a^{\dagger}\left|K_{1}\right| a^{\dagger}>^{n} \Omega\right\|^{2}$.

Here we shall briefly summarize properties of an infinite determinant of a trace class operator.

Proposition 3.6 : Let $A, B \in I_{2}(\mathcal{H})$. Then:

(1) $\operatorname{det}\left(I+z A^{*} A\right)$ is a holomolphic function in $z \in \mathbb{C}$ and has no zero points in $|z|<\|A\|^{-2}$.

(2) For $z \in \mathbb{C}$,

$$
\left|\frac{d^{n}}{d z^{n}} \operatorname{det}\left(I+z A^{*} A\right)-\frac{d^{n}}{d z^{n}} \operatorname{det}\left(I+z B^{*} B\right)\right| \leq\left\|A^{*} A-B^{*} B\right\|_{1} C_{n}\left(\|A\|_{2}^{2},\|B\|_{2}^{2},|z|\right) .
$$

where $C_{n}(\cdot, \cdot, \cdot)$ is a continous function on $\mathbb{R} \times \mathbb{R} \times \mathbb{R}$.

Proof: From $\S$ XIII 17, Lemma 4 in Ref.10, it follows (1) and

$$
\left|\operatorname{det}\left(I+z A^{*} A\right)-\operatorname{det}\left(I+z B^{*} B\right)\right| \leq\left\|A^{*} A-B^{*} B\right\|_{T R} C_{0}\left(\|A\|_{H S}^{2},\|B\|_{H S}^{2},|z|\right),
$$

where $C_{0}(x, y, z)=z e^{z(x+y)+1}$. Then for $n \geq 1$, we have

$$
\begin{aligned}
& \left|\frac{d^{n}}{d z^{n}} \operatorname{det}\left(I+z A^{*} A\right)-\frac{d^{n}}{d z^{n}} \operatorname{det}\left(I+z B^{*} B\right)\right| \\
& \leq \frac{n !}{2 \pi} \int_{|w-z|=r}\left|\frac{\operatorname{det}\left(I+\omega A^{*} A\right)-\operatorname{det}\left(I+\omega B^{*} B\right)}{(\omega-z)^{n+1}}\right| d \omega \\
& \leq \frac{n !}{r^{n+1}} C_{0}\left(\|A\|_{H S}^{2},\|B\|_{H S}^{2},|z|+r\right)\left\|A^{*} A-B^{*} B\right\|_{T R},
\end{aligned}
$$

where $r>0$. Thus (2) follows.

By Proposition 3.6 (1), we can define a family of holomorphic functions on the open set $\dot{\vartheta}$ containing 1 with parameter $k \geq 0$ by:

$$
\begin{aligned}
& \left\{F_{\kappa}(z, k)\right\}_{0<\kappa<\infty}=\left\{\sum_{n=0}^{\infty} n^{k} b_{n}(\kappa) z^{n}\right\}_{0<\kappa<\infty}, \\
& F(z, \dot{k})=\sum_{n=0}^{\infty} n^{k} b_{n} z^{n} .
\end{aligned}
$$

Lemma 3.7 : For any $k \geq 0$, and $z \in \dot{\vartheta}$, the following limit exists

$$
\lim _{\kappa \rightarrow \infty} F_{\kappa}(z, k)=F(z, k) .
$$

Proof: We use induction on $k$. Let fix $z \in \dot{\vartheta}$ throughout the proof. In the case $k=0$, by (3.17) and Proposition 3.6, we can see

$$
\left|F_{\kappa}(z, 0)^{-2}-F(z, 0)^{-2}\right| \leq\left(\left\|K_{1}^{*}(\kappa) K_{1}(\kappa)-K_{1}^{*} K_{1}\right\|_{T R}\right) \times C_{0}\left(\left\|K_{1}(\kappa)\right\|_{H S}^{2},\left\|K_{1}\right\|_{H S}^{2},|z|\right) .
$$


Hence the r.h.s.of (3.18) converges to 0 as $\kappa \rightarrow \infty$. Thus lemma holds for $k=0$.

Assuming that the lemma is true for all $k \leq l$ for some $l \geq 0$, we can see that for $l+1$,

$$
F_{\kappa}(z, l+1)=\left\{\frac{d^{l+1}}{d z^{l+1}}\left(\operatorname{det}\left(I-z K^{*}(\kappa) K(\kappa)\right)^{-\frac{1}{2}}\right)\right\} z^{l+1}+\sum_{n=0}^{\infty} P(n, l) a_{n}(\kappa) z^{n}
$$

where $P(n, l)=n^{l+1}-n(n-1) \ldots(n-l)$. We see that a first term of the r.h.s of Eq.(3.19) is a sum of finite number of terms, each of which is a product of

$$
\operatorname{det}\left(I-z K_{1}^{*}(\kappa) K_{1}(\kappa)\right)^{-\frac{2 l+3}{2}}, \frac{d^{j}}{d z^{j}} \operatorname{det}\left(I-z K_{1}^{*}(\kappa) K_{1}(\kappa)\right), j=1, \ldots, l .
$$

It follows from Proposition 3.6 (2) that the limits of the both terms of Eq.(3.20) exists with

$$
\begin{aligned}
& \lim _{\kappa \rightarrow \infty} \operatorname{det}\left(I-z K_{1}^{*}(\kappa) K_{1}(\kappa)\right)^{-\frac{2 l+3}{2}}=\operatorname{det}\left(I-z K_{1}^{*} K_{1}\right)^{-\frac{2 l+3}{2}}, \\
& \lim _{\kappa \rightarrow \infty} \frac{d}{d z^{j}} \operatorname{det}\left(I-z K_{1}^{*}(\kappa) K_{1}(\kappa)\right)=\frac{d}{d z^{j}} \operatorname{det}\left(I-z K_{1}^{*} K_{1}\right) .
\end{aligned}
$$

On the other hand, by the induction hypothesis, the limit of the second term of the r.h.s of Eq.(3.19) exists with

$$
\lim _{\kappa \rightarrow \infty} \sum_{n=0}^{\infty} P(n, l) a_{n}(\kappa) z^{n}=\sum_{n=0}^{\infty} P(n, l) a_{n} z^{n} .
$$

Thus we have completed the proof of Lemma 3.7.

In what follows, for the sake of brevity, we denote $\Omega\left(K_{1}(\kappa)\right)$ by $\Omega(\kappa), 0<\kappa<\infty$, and $\Omega\left(K_{1}\right)=\Omega(\infty)$. Lemma 3.7 immediately gives the following corollary.

Corollary $3.8:$ For any $k \geq 0$, the following limit exists:

$$
\lim _{\kappa \rightarrow \infty}\left\|\mathrm{N}^{k} \Omega(\kappa)\right\|=\left\|\mathrm{N}^{k} \Omega(\infty)\right\|
$$

Proof: Because of Proposition 1.1 (3) and Lemma 3.7, we have

$$
\lim _{\kappa \rightarrow \infty}\left\|\mathrm{N}^{k} \Omega(\kappa)\right\|^{2}=\lim _{\kappa \rightarrow \infty} F_{\kappa}(1,2 k)=F(1,2 k)=\left\|\mathrm{N}^{k} \Omega(\infty)\right\|^{2} .
$$

Thus Corollary 3.8 follows.

Lemma 3.9 : For any $k \geq 0$, the following limit exists:

$$
s-\lim _{\kappa \rightarrow \infty} \mathrm{N}^{k} \Omega(\kappa)=\mathrm{N}^{k} \Omega(\infty) .
$$


Proof: We begin with showing the existence of the weak limit of $\Omega(\kappa)$ as $\kappa \rightarrow \infty$. Let $\Phi$ be of the form $\Phi=a^{\dagger}\left(f_{1}\right) \ldots a^{\dagger}\left(f_{n}\right) \Omega \in \mathcal{D}$. If $n$ is odd, then $\left(\mathrm{N}^{k} \Omega(\kappa), \Phi\right)=0$. Hence it suffices to consider the case $n=2 m(m \in \mathbb{N})$. Then we see that

$$
\left(\mathrm{N}^{k} \Omega(\kappa), \Phi\right)=(2 m)^{k}\left(-\frac{1}{2}\right)^{m} \frac{1}{m !}\left(\Omega,<a\left|K_{1}^{*}(\kappa)\right| a>^{m} \Phi\right) .
$$

It follows from Eq.(2.7) that $\left\langle a\left|K_{1}^{*}(\kappa)\right| a\right\rangle^{m} \Phi$ can be represented as a finite sum of terms, each of which is a product of $\left(f_{i}, K_{1}^{*}(\kappa) f_{j}\right)(i, j=1, \ldots, n)$. Hence one can see that in strong topology, $<a\left|K_{1}^{*}(\kappa)\right| a>\Phi \rightarrow<a\left|K_{1}^{*}\right| a>\Phi$ as $\kappa \rightarrow \infty$. Thus we get

$$
\left(\Omega,<a\left|K_{1}^{*}(\kappa)\right| a>^{m} \Phi\right) \longrightarrow\left(\Omega,<a\left|K_{1}^{*}\right| a>^{m} \Phi\right) \quad(\kappa \rightarrow \infty) .
$$

Since $\mathcal{D}$ is dense and Corollary 3.8 gives that $\left\|N^{k} \Omega(\kappa)\right\|$ is bounded in $\kappa$, we have

$$
w-\lim _{\kappa \rightarrow \infty} \mathrm{N}^{k} \Omega(\kappa)=\mathrm{N}^{k} \Omega(\infty) .
$$

Then Lemma 3.9 follows from Corollary 3.8 and Eq.(3.21).

Lemma 3.10 : The strong limit of the unitary operator $U(\kappa)$ exists with

$$
s-\lim _{\kappa \rightarrow \infty} \mathrm{U}(\kappa)=\mathrm{U}
$$

Proof: It suffices to prove Eq.(3.22) on a dense subspace $\mathcal{D}$. Let $\Phi=a^{\dagger}\left(f_{1}\right) \ldots a^{\dagger}\left(f_{n}\right) \Omega \in \mathcal{D}$. By Theorem 3.3 , we have

$$
\begin{aligned}
\|\mathrm{U}(\kappa) \Phi-\mathrm{U} \Phi\| & =\left\|b_{\kappa}^{\dagger}\left(f_{1}\right) \ldots b_{\kappa}^{\dagger}\left(f_{n}\right) \Omega(\kappa)-b^{\dagger}\left(f_{1}\right) \ldots b^{\dagger}\left(f_{n}\right) \Omega(\infty)\right\| \\
& =\left\|\Pi_{j=1}^{n}\left\{a^{\dagger}\left(\bar{S}(\kappa) f_{j}\right)+a\left(\bar{T}(\kappa) f_{j}\right)\right\} \Omega(\kappa)-\Pi_{j=1}^{n}\left\{a^{\dagger}\left(\bar{S} f_{j}\right)+a\left(\bar{T} f_{j}\right)\right\} \Omega(\infty)\right\| \\
& \leq \sum^{\text {finite }}\left\|a^{\sharp 1}\left(X_{1, \kappa} f_{1}\right) . . a^{\sharp_{n}}\left(X_{n, \kappa} f_{n}\right) \Omega(\kappa)-a^{\sharp 1}\left(X_{1} f_{1}\right) . . a^{\ell_{n}}\left(X_{n} f_{n}\right) \Omega(\infty)\right\|(3.23)
\end{aligned}
$$

where $a^{\sharp j}$ means $a$ or $a^{\dagger}, X_{j, \kappa}, \bar{S}(\kappa)$ or $\bar{T}(\kappa)(0<\kappa<\infty)$, and $X_{j}, \bar{S}$ or $\bar{T}, j=1, \ldots, n$. Since the summation in the last inequality (3.23) is taken over a finite number of terms, we need only to prove that each term goes to zero as $\kappa \rightarrow \infty$. We have

$$
\begin{aligned}
& \left\|a^{\sharp_{1}}\left(X_{1, \kappa} f_{1}\right) \ldots a^{l_{n}}\left(X_{n, \kappa} f_{n}\right) \Omega(\kappa)-a^{h_{1}}\left(X_{1} f_{1}\right) \ldots a^{\sharp_{n}}\left(X_{n} f_{n}\right) \Omega(\infty)\right\| \\
& \leq \sum_{j=1}^{n}\left\|a^{\sharp_{1}}\left(X_{1, \kappa} f_{1}\right) \ldots a^{\|_{j}}\left(\left(X_{j, \kappa}-X_{j}\right) f_{j}\right) \ldots a^{\sharp_{n}}\left(X_{n} f_{n}\right) \Omega(\infty)\right\| \\
& \quad+\left\|a^{\sharp_{1}}\left(X_{1, \kappa} f_{1}\right) \ldots a^{\sharp_{n}}\left(X_{n, \kappa} f_{n}\right)(\Omega(\kappa)-\Omega(\infty))\right\| .
\end{aligned}
$$


The two terms in (3.24) can be estimated as follows:

$$
\lim _{\kappa \rightarrow \infty}\left\|a^{\sharp_{1}}\left(X_{1, \kappa} f_{1}\right) \ldots a^{\sharp_{j}}\left(\left(X_{j, \kappa}-X_{j}\right) f_{j}\right) \ldots a^{\sharp_{n}}\left(X_{n} f_{n}\right) \ldots \Omega(\infty)\right\|=0, j=1, \ldots, n .
$$

Since $\Omega(\infty)$ is in $\mathcal{D}_{\infty}$, by making use of (2.1) and Eq.(2.2) again, we have

$$
\begin{aligned}
& \quad\left\|a^{\sharp_{1}}\left(X_{1, \kappa} f_{1}\right) \ldots a^{\sharp_{n}}\left(X_{n, \kappa} f_{n}\right)(\Omega(\kappa)-\Omega(\infty))\right\| \\
& \begin{array}{l}
\leq\left\|X_{1, \kappa}\right\|\left(n\left\|a^{\sharp_{2}}\left(X_{2, \kappa} f_{2}\right) \ldots a^{\sharp_{n}}\left(X_{n, \kappa} f_{n}\right)(\Omega(\kappa)-\Omega(\infty))\right\|\right. \\
\left.\quad \quad \quad\left\|a^{\sharp_{2}}\left(X_{2, \kappa} f_{2}\right) \ldots a^{\sharp_{n}}\left(X_{n, \kappa} f_{n}\right)(\mathrm{N}+1)(\Omega(\kappa)-\Omega(\infty))\right\|\right)
\end{array} \\
& \quad \ldots \ldots . \\
& \leq \Pi_{l=1}^{n}\left\|X_{l, \kappa} f_{l}\right\| \sum_{j=0}^{n}\left(\begin{array}{c}
n \\
j
\end{array}\right) n^{j}\left\|(\mathrm{~N}+1)^{j}(\Omega(\kappa)-\Omega(\infty))\right\|
\end{aligned}
$$

It follows from Lemma 3.9 that

$$
\lim _{\kappa \rightarrow \infty} \sum_{j=0}^{n}\left(\begin{array}{l}
n \\
j
\end{array}\right) n^{j}\left\|(\mathrm{~N}+1)^{j}(\Omega(\kappa)-\Omega(\infty))\right\|=0 .
$$

Thus Eq.(3.22) follows.

We define the operators $\pi$ and $\pi(\kappa)$ in $\mathcal{F}(\mathcal{H})$ by

$$
\begin{aligned}
& \pi(\kappa)=i\left\{b_{\kappa}(L(\kappa))-b_{\kappa}^{\dagger}(\overline{L(\kappa)})\right\}, 0<\kappa<\infty, \\
& \pi=i\left\{b(L)-b^{\dagger}(\bar{L})\right\} .
\end{aligned}
$$

It is easy to see that $\pi(\kappa)$ and $\pi$ are essentially self-adjoint operators on $\mathcal{F}_{0}(\mathcal{H})$ for $0<\kappa<\infty$. We denote their self-adjoint extentions by the same symbols. Then by Lemma 3.3, the Displacement operators $\mathbf{S}(\kappa)$ and $\mathbf{S}$ have the forms

$$
\begin{aligned}
& \mathbf{S}(\kappa)=\exp (-i \pi(\kappa)), 0<\kappa<\infty \\
& \mathbf{S}=\exp (-i \pi)
\end{aligned}
$$

By conditions (A.2) and (A.4), one can immediately see that

$$
s-\lim _{\kappa \rightarrow \infty} \mathbf{S}(\kappa)=\mathbf{S}
$$

Now we are ready to prove the main theorem in this subsection. 
Theorem 3.11 : The family of unitary operators $\{\mathcal{U}(\kappa)\}_{0<\kappa<\infty}$ has strong limit as $\kappa \rightarrow \infty$;

$$
s-\lim _{\kappa \rightarrow \infty} \mathcal{U}(\kappa)=\mathcal{U}
$$

Proof: Since $\mathcal{U}(\kappa)=\mathrm{U}(\kappa) \mathrm{S}(\kappa)$, Eq.(3.26) follows from Lemma 3.10 and Eq.(3.25).

\section{ASYMPTOTIC BEHAVIOR FOR A CLASS OF SELF-ADJOINT OPERATORS}

In this section, we shall briefly summarize the asymptotic theory for a family of self-adjoint operators. This kind of work has been done in many literatures. In paticular, we refer to Ref.I and use the same notations as those in Ref.1.

Let $\mathcal{K}$ be a complex separable Hilbert space and set

$$
\mathcal{X}=\mathcal{H} \otimes \mathcal{K}
$$

Suppose that $A$ and $B$ are nonnegative self-adjoint operators in $\mathcal{H}$ and $\mathcal{K}$, respectively, and $K \operatorname{er} B \neq$ $\{0\}$. Let $P_{0}$ denote the projection from $\mathcal{K}$ to $\operatorname{Ker} B$. Let $\left\{C_{\kappa}\right\}_{\kappa>0}$ be a family of symmetric operators in $\mathcal{X}$ and $F_{j}, j=1,2$ real valued functions on $\mathbb{R}$ which satsfy the following conditions:

$(\mathrm{C}-1)$ : For all $\kappa>0, D(A \otimes I) \subset D\left(C_{\kappa}\right)$ and $C_{\kappa}(A \otimes I+\lambda)^{-1}$ is a bounded operator for all $\lambda>0$ on $\mathcal{X}$ with

$$
\lim _{\lambda \rightarrow \infty}\left\|C_{\kappa}(A \otimes I+\lambda)^{-1}\right\|=0 .
$$

This convergence is uniform in $\kappa \geq \kappa_{0}$ for some $\kappa_{0}>0$.

$(\mathrm{C}-2)$ : There exists a symmetric operator $C$ in $\mathcal{X}$ such that $D(A \otimes I) \subset D(C)$,

$$
s-\lim _{\kappa \rightarrow \infty} C_{\kappa}(A \otimes I+\lambda)^{-1}=C(A \otimes I+\lambda)^{-1} .
$$

$(\mathrm{C}-3)$ : For $j=1,2$, the limit $\lim _{\kappa \rightarrow \infty} F_{j}(\kappa)=F_{j}<\infty$, exists.

We define the operators in $\mathcal{X}, H_{0, \kappa}$ and $H_{\kappa}$, by

$$
\begin{aligned}
H_{0, \kappa} & =F_{1}(\kappa) A \otimes I+\kappa I \otimes B+F_{2}(\kappa), \\
H_{\kappa} & =H_{0, \kappa}+C_{\kappa} .
\end{aligned}
$$

Lemma 4.1 : We put $D(A \otimes I) \cap D(I \otimes B)=D_{A B}$. Then $H_{\kappa}$ is self-adjoint operator on $D_{A B}$, bounded from below and essentially self-adjoint on any core of $H_{0, \kappa}$ with $\kappa \geq \kappa_{0}$. 
Proof: With a little modification of Theorem 3.1 in Ref.1, one can easily prove Lemma 4.1.

We say that a densely defined linear operator $S$ in $\mathcal{X}$ is in $\mathbb{E}(\mathcal{X})$ if and only if there exist subspaces $D_{\mathcal{H}}(S)$ and $D_{\mathcal{K}}(S)$ dense in $\mathcal{H}$ and $\mathcal{K}$, respectively, such that

$$
D_{\mathcal{H}}(S) \widehat{\otimes} D_{\mathcal{K}}(S) \subset D(S)
$$

where $\widehat{\otimes}$ means algebraic tensor product. For $S \in \mathbb{E}(\mathcal{X}), f \in \mathcal{K}$ and $g \in D_{\mathcal{K}}(S)$, we can define the partial expectation $E_{f, g}(S)$ which is a densely defined linear operator in $\mathcal{H}$ such that

$$
\begin{aligned}
& D\left(E_{f, g}(S)\right)=D_{\mathcal{H}}(S) \\
& \left(u, E_{f, g}(S) v\right)_{\mathcal{H}}=(u \otimes f, S(v \otimes g))_{\mathcal{X}}, u \in \mathcal{H}, v \in D_{\mathcal{H}}(S) .
\end{aligned}
$$

Suppose that $\operatorname{Ker} B=\left\{\alpha f_{0} \mid \alpha \in \mathbb{C}\right\}$ with some $f_{0}$ satisfying $\left\|f_{0}\right\|=1$. Then we define an operator $H_{\text {eff }}$ in $\mathcal{H}$ by

$$
H_{e f f}=F_{1} A+E_{\text {eff }}(C)+F_{2}, \text { where } E_{\text {eff }}(C)=E_{f, f}(C) .
$$

Lemma 4.2 : Let $C \in \mathbb{E}(\mathcal{X})$ with $D_{\mathcal{K}}(C) \subset \operatorname{Ker} B$, and $\operatorname{Ker} B=\left\{\alpha f_{0} \mid \alpha \in \mathbb{C}\right\}$. Then, for all $z \in C$ with $\operatorname{Im} z \neq 0$ or $z<0$ with $|z|$ sufficiently large,

$$
\lim _{\kappa \rightarrow \infty}\left(H_{\kappa}+z\right)^{-1}=\left(H_{e f f}+z\right)^{-1} \otimes P_{0} .
$$

Proof: See Ref.1.

\section{A MODEL IN QUANTUM ELECTRODYNAMICS}

\subsection{Definition and some fundamental facts}

We consider a model which describes a quantum system of one electron atom interacting with a quantized radiation field. For a mathematical generality, we consider the situation where the electron moves in $\mathbb{R}^{d}$ and the radiation field is over $\mathbb{R}^{d}(d \geq 3)$. We shall denote by $\hbar$ (resp.m, $c, e$ ) the Planck constant devided by $2 \pi$ (resp. the mass of an electron, the speed of light and elementary charge). We quantize the free classical radiation field in the Colomb gauge; the Hilbert space of state vectors for the quantised radiation field is then defined by the Boson Fock space

$$
\mathcal{F}^{E M} \equiv \bigoplus_{n=0}^{\infty} \otimes_{s}^{n} \mathcal{W}
$$


over the Hilbert space

$$
\mathcal{W}=\underbrace{L^{2}\left(\mathbb{R}^{d}\right) \oplus \ldots \oplus L^{2}\left(\mathbb{R}^{d}\right)}_{\text {d-1 times }}
$$

which denotes the one photon space. We denote by $\mathcal{F}_{0}^{E M}$ the finite particle space in $\mathcal{F}^{E M}$. For $f \in \mathcal{W}$ we denote a creation operator in $\mathcal{F}^{E M}$ by $a^{\dagger}(f)$, and define an annihilation operator $a(f)$ in $\mathcal{F}^{E M}$ in the same way as in the case of the abstract Boson Fock space in Section III. For $f \in L^{2}\left(\mathbb{R}^{d}\right)$, we put

$$
f_{r}=(0, \ldots, 0, f, 0, \ldots, 0) \in \mathcal{W} \quad(1 \leq r \leq d-1)
$$

(the $\mathrm{r}$-th component is $f$ and the others are zero) and define $a^{\sharp(r)}(f)$ by $a^{\sharp}\left(f_{r}\right), f \in L^{2}(\mathbb{R})$. We denote by $a^{\sharp(r)}(k)$ the formal distribution kernel:

$$
a^{\sharp(r)}(f)=\int a^{\sharp(r)}(k) f(k) d k .
$$

The operator $a^{\sharp(r)}(f)\left(f \in L^{2}\left(\mathbb{R}^{d}\right)\right)$ is well definded on $\mathcal{F}_{0}^{E M}$ and leaves it invariant satisfying

$$
\begin{aligned}
& {\left[a^{(r)}(f), a^{(s)}(g)\right]=\left[a^{\dagger(r)}(f), a^{\dagger(s)}(g)\right]=0,} \\
& {\left[a^{(r)}(f), a^{\dagger(s)}(g)\right]=\delta_{r s}(\bar{f}, g) f, g \in L^{2}\left(\mathbb{R}^{d}\right), 1 \leq r, s \leq d .}
\end{aligned}
$$

Let $e^{r}(\cdot): \mathbb{R}^{d} \longrightarrow \mathbb{R}^{d}(1 \leq r \leq d-1)$, be a mesurable function such that

$$
(1) k \cdot e^{r}(k)=0, \quad(2) e^{r}(k) \cdot e^{s}(k)=\delta_{r s}, \quad a . e . k \in \mathbb{R}^{d}
$$

The vector $e^{r}$ surves as a polarization vector of photon. We shall denote the $\mu$ th component of $e^{r}$ by $e_{\mu}^{\tau},(\mu=1, \ldots, d)$. Then the time-zero radiation field and the cojugate momentum are defined by

$$
\begin{aligned}
& A_{\mu}(x, f)=\frac{1}{\sqrt{2}} \sum_{r=1}^{d-1} \int d k \frac{\sqrt{\hbar}}{\sqrt{c \omega(k)}} e_{\mu}^{r}\left\{\hat{f}(k) e^{-i k x} a^{\dagger(r)}(k)+\tilde{\hat{f}}(k) e^{i k x} a^{(r)}(k)\right\} \\
& \Pi_{\mu}(x, f)=\frac{i}{\sqrt{2}} \sum_{r=1}^{d-1} \int d k \sqrt{\hbar} \sqrt{c \omega(k)} e_{\mu}^{r}(k)\left\{\hat{f}(k) e^{i k x} a^{\dagger(r)}(k)-\tilde{\hat{f}}(k) e^{-i k x} a^{(r)}(k)\right\}
\end{aligned}
$$

where $\omega(k)=|k|, i=\sqrt{-1}, \tilde{f}(k)=f(-k)$ and $\hat{f}$ is the Fourier transform defined by

$$
\hat{f}(k)=\frac{1}{(2 \pi)^{\frac{d}{2}}} \int_{\mathbb{R}^{d}} d x f(x) e^{-i k \cdot x} .
$$


The function $\omega(k)$ is regaded as the energy of a free photon with momentum $k$. For the sake of simplicity, we write $A_{\mu}(0, f)$ and $\Pi_{\mu}(0, f)$ as $A_{\mu}(f)$ and $\Pi_{\mu}(f)$ respectively. Let

$$
\widehat{\omega}=\underbrace{\omega \oplus \ldots \oplus \omega}_{\mathrm{d}-1} .
$$

The free Hamiltonian of the quantized radiation field is defined by

$$
H^{E M}=\hbar c d \Gamma(\widehat{\omega})
$$

For each $\alpha \in \mathbb{R}$, we define a Hilbert space $M_{\alpha}$ by

$$
M_{\alpha}=\left\{\left.f\left|\|f\|_{\alpha}^{2} \equiv \int_{\mathbb{R}^{d}} \omega(k)^{2 \alpha}\right| f(k)\right|^{2} d k<\infty\right\}
$$

The field operators $A_{\mu}, \Pi_{\mu}, H^{E M}$ satisfy the following commutation relations on $\mathcal{F}_{0}^{E M}$,

$$
\begin{array}{rlrl}
{\left[A_{\mu}(f), A_{\nu}(g)\right]} & =0 & & \hat{f}, \hat{g} \in M_{-\frac{1}{2}}, \\
{\left[\Pi_{\mu}(f), \Pi_{\nu}(g)\right]=0} & & \hat{f}, \hat{g} \in M_{\frac{1}{2}} \\
{\left[A_{\mu}(f), \Pi_{\nu}(g)\right]} & =i \hbar\left(d_{\mu \nu} \hat{\bar{f}}, \hat{g}\right) & & \hat{f}, \hat{g} \in M_{-\frac{1}{2}} \cap M_{\frac{1}{2}},
\end{array}
$$

and on $D\left(H^{E M \frac{3}{2}}\right)$,

$$
\begin{array}{ll}
{\left[H^{E M}, A_{\mu}(f)\right]=-i \hbar \Pi_{\mu}(f)} & \hat{f} \in M_{-\frac{1}{2}} \cap M_{\frac{1}{2}}, \\
{\left[H^{E M}, \Pi_{\mu}(f)\right]=i \hbar c^{2} A_{\mu}(-\Delta f)} & \hat{f} \in M_{\frac{3}{3}} \cap M_{\frac{1}{2}},
\end{array}
$$

where $\Delta$ is the Laplacian in the generalized sense and $d_{\mu \nu}(k)=e_{\mu}^{r}(k) e_{\nu}^{r}(k)$ (summation over repeated indices is understood).

The Hilbert space $\mathcal{F}$ of state vectors for the interacting system of the one electron atom and the quantized radiation field is of the form:

$$
\mathcal{F}=L^{2}\left(\mathbb{R}^{d}\right) \otimes \mathcal{F}^{E M}
$$

The electron momentum operator is given by the differential operator in the generalized sense $\mathrm{p}_{j}=-i \hbar \partial / \partial x_{j},(j=1, \ldots, d), x=\left(x_{1}, \ldots, x_{d}\right) \in \mathbb{R}^{d}$,

$$
\mathrm{p}=\left(\mathrm{p}_{1}, \ldots, \mathrm{p}_{d}\right)
$$

In order to define the interaction between the one electron atom and the radiation field as an operator in $\mathcal{F}$, we have to introduce a cut off for photon momenta: let $\rho \in \mathcal{S}\left(\mathbb{R}^{d}\right)\left(\mathcal{S}\left(\mathbb{R}^{d}\right)\right.$ is the set 
of infinitely many times differentiable rapidly decreasing functions on $\mathbb{R}^{d}$ ) be positive and rotation invariant:

$$
\hat{\rho}(k)>0 \text { for } a . e . k \in \mathbb{R}^{d} \text { and } \hat{\rho}(k)=\hat{\rho}(|k|) \text {. }
$$

Moreover we suppose

$$
\int_{\mathbb{R}^{d}} \frac{\hat{\rho}^{2}(k)}{\omega(k)^{3}} d k<\infty .
$$

Here $\hat{\rho}$ surves as a cut-off for photon momenta. Then the free Hamiltonian of the coupled system of the one electron atom and the quantized radiation field with the full minimal interaction and the cut off $\hat{\rho}$ is defined by

$$
H=\frac{1}{2 m}(\mathbf{p} \otimes I+e A(x, \rho))^{2}+I \otimes H^{E M} .
$$

In this paper, however, we take as the free Hamiltonian of the coupled system a version of $H$ simplified by the dipole approximation, which replaces the term $A(x, \rho)$ with $A(\rho)$. Moreover, introducing a parameter $0 \leq \epsilon \leq 1$, we define the operator $H_{\epsilon}$ in $\mathcal{F}$ by

$$
H_{\epsilon}=\frac{1}{2 m}\left(\mathbf{p}^{2} \otimes I+2 e \mathbf{p}_{\mu} \otimes A_{\mu}(\rho)+\epsilon e^{2} I \otimes A^{2}(\rho)\right)+I \otimes H^{E M}
$$

The operator $H_{\epsilon}$ is the main object to be analyzed in this paper. In the case $\epsilon=0$, it corresponds to a simpler model which has been considerd in Ref.1, and in the case $\epsilon=1$, to the model $H$ with the dipole approximation. We investigate an asymptotic behavior of $H_{\epsilon}$ and compare it with that of $H_{0}(\epsilon=0)$.

Proposition 5.1 : The operator $H_{\epsilon}(0 \leq \epsilon \leq 1)$ is self-adjoint on $D\left(\mathrm{p}^{2} \otimes I\right) \cap D\left(I \otimes H^{E M}\right)$ and bounded from below. Furthermore it is essentially self-adjoint on any core of $\mathrm{p}^{2} \otimes I+I \otimes H^{E M}$. Proof: See Ref.1.

It is well known that there exists a unitary operator $\Theta$ on $\mathcal{F}$ such that

$$
\Theta \mathcal{F}=L^{2}\left(\mathbb{R}^{d} ; \mathcal{F}^{E M}\right)
$$

The r.h.s. of Eq.(5.1) is the set of $\mathcal{F}^{E M}$-valued $L^{2}$ functions on $\mathbb{R}^{d}$. Let $F$ be the Fourier transfom from $L^{2}\left(\mathbb{R}^{d}\right)$ onto itself and set $\Theta(F \otimes I)=\mathrm{F}$. Then $\mathrm{F}$ is the unitary operator on $\mathcal{F}$ and set

$$
\mathrm{F} \mathcal{F}=\widehat{L}^{2}\left(\mathbb{R}^{d} ; \mathcal{F}^{E M}\right)
$$


It is not hard to see that the operator $\mathrm{FH}_{\epsilon} \mathrm{F}^{-1}$ in $\widehat{L}^{2}\left(\mathbb{R}^{d} ; \mathcal{F}^{E M}\right)$ is decompsable: (Ref.11)

$$
\mathrm{F} H_{\epsilon} \mathrm{F}^{-1}=\int_{\mathbb{R}^{d}}^{\oplus} H_{\epsilon}(p) d p
$$

where

$$
H_{\epsilon}(p)=\frac{1}{2 m} \hbar^{2} p^{2}+\frac{e}{m} p_{\mu} \cdot A_{\mu}(\rho)+\epsilon \frac{e^{2}}{2 m} A^{2}(\rho)+H^{E M}, \quad\left(p \in \mathbb{R}^{d}\right) .
$$

Theorem 5.2 : For every $p \in \mathbb{R}^{d}$, the operator $H_{\epsilon}(p)(0 \leq \epsilon \leq 1)$ is self-adjoint on $D\left(H^{E M}\right)$ and bounded from below. Furthermore it is essentially self-adjoint on any core of $H^{E M}$.

Proof: See Ref.11.

\subsection{The Heisenberg field}

In this subsection we shall prove a unitary equivalence of $H_{\epsilon}(p)$ and $H^{E M}$, and derive an explicit form of the coresponding unitary operator by making use of abstract results in Section III. In order to do this, we need to solve the Heisenberg equation of field operators explicitly. The Heisenberg field $A_{\mu}(f, t)\left(f \in L^{2}\left(\mathbb{R}^{d}\right), t \in \mathbb{R}\right)$ is definded by a solution of the Heisenberg equation:

$$
\begin{aligned}
& \frac{d}{d t} A_{\mu}(f, t)=\frac{i}{\hbar}\left[H_{\epsilon}(p), A_{\mu}(f, t)\right], \\
& A_{\mu}(f, 0)=A_{\mu}(f) .
\end{aligned}
$$

The canonical cojugate momentum is defined by a solution of the following equation:

$$
\begin{aligned}
& \frac{d}{d t} \Pi_{\mu}(f, t)=\frac{i}{\hbar}\left[H_{\epsilon}(p), \Pi_{\mu}(f, t)\right], \\
& \Pi_{\mu}(f, 0)=\Pi_{\mu}(f) .
\end{aligned}
$$

In the case where $\epsilon=1$, the equation Eq.(5.2) has been solved in Ref.11. Mimicking the method used in Refs.11,12 and 13, we shall solve Eq.(5.2). Any solution of the Heisenberg equation $A_{\mu}(f, t)$ satisfies the following formal equation:

$$
\left(\frac{1}{\hbar} \frac{\partial^{2}}{\partial t^{2}}-c^{2} \Delta\right) A_{\mu}(x, t)=-\frac{e}{m} \rho_{\mu \nu}\left(\hbar p_{\nu}+\epsilon e A_{\nu}(\rho, t)\right),
$$

where

$$
\rho_{\mu \nu}=(2 \pi)^{-\frac{d}{2}} \int d_{\mu \nu}(k) \widehat{p}(k) e^{i k x} d k
$$


and $A_{\mu}(x, t)$ is a formal kernel of $A_{\mu}(f, t)$

$$
\int A_{\mu}(x, t) f(x) d x=A_{\mu}(f, t)
$$

We define

$$
D_{\epsilon}(z)=m-\epsilon \frac{e^{2}}{c^{2}} \frac{d-1}{d} \int_{\mathbb{R}^{d}} \frac{\hat{\rho}(k)^{2}}{z-k^{2}} d k, \quad z \in \mathbb{C} \backslash[0, \infty) .
$$

The function $D_{\epsilon}(z)$ satisfies the following:

\section{Lemma 5.3 :}

(1) The function $D_{\epsilon}$ is analytic in $\mathbb{C} \backslash[0, \infty)$ and has no zero points in $\mathbb{C} \backslash[0, \infty)$.

(2) For $s \in[0, \infty), D_{\epsilon \pm}(s)=\lim _{h \rightarrow 0} D_{\epsilon}(s \pm i h)$ exists and $\lim _{s \rightarrow \infty} D_{\epsilon \pm}(s)=m$

(3) There exists $d_{m}>0$ such that $\left|D_{\epsilon+}(s)\right|>d_{m}, \quad s \in[0, \infty)$.

(4) For all $s \in[0, \infty)$,

$$
D_{\epsilon+}(s)-D_{\epsilon-}(s)=2 \pi i \epsilon \frac{e^{2}}{c^{2}}\left(\frac{d-1}{d} V_{d}\right) \hat{\rho}^{2}(\sqrt{s}) s^{\frac{d}{2}-1},
$$

where $\mathrm{V}_{d}$ is a volume of the $(d-1)$-sphere $\mathrm{S}_{d-1}$. It is given by $\mathrm{V}_{d}=2 \pi^{\frac{d}{2}} / \Gamma\left(\frac{d}{2}\right)(\Gamma(z)$ is the gamma function).

Proof: An elementary exercise. See Refs.11,12 and 13.

We define the opeator $G_{h}(h>0)$ by

$$
\left(G_{h} f\right)(k)=\int_{\mathbb{R}^{d}} \frac{f\left(k^{\prime}\right)}{\left(k^{2}-k^{\prime 2}+i h\right)\left(k k^{\prime}\right)^{\frac{d}{2}-1}} d k^{\prime} .
$$

It is not so hard to see that $G_{h}$ is a bounded linear operator on $L^{2}\left(\mathbb{R}^{d}\right)$ and the strong limit $\lim _{h \rightarrow 0} G_{h} \equiv G$ exists. Furthermore $G$ is skew symmetric $\left(G^{*}=-G\right)$ and also the bounded operator on $M_{1-\frac{d}{2}}$. Because of Lemma 5.3 (3), we can define

$$
Q_{\epsilon \pm}(k)=\frac{e \hat{\rho}(k)}{D_{\epsilon \pm}\left(k^{2}\right)}, k \in \mathbb{R}^{d}
$$

For simplisity, we put

$$
Q_{\epsilon+}=Q
$$

We set

$$
T_{\mu \nu} f=\delta_{\mu \nu} f+\epsilon \frac{e}{c^{2}} Q \omega^{\frac{d}{2}-1} G \omega^{\frac{d}{2}-1} d_{\mu \nu} \hat{\rho} f, \quad(1 \leq \mu, \nu \leq d) .
$$


Lemma 5.4 : Throughtout this lemma, we suppose that $m>0$ or $m<0$, and denote the Heaviside function by $\theta$;

$$
\theta(m)= \begin{cases}1, & m>0 \\ 0, & m<0\end{cases}
$$

In the case where $m<0$, the function $D(\cdot)$ has a unique zero point in $(-\infty, 0)$. We denote it $-E^{2}(E>0) . \gamma$ is defined only in the case $m<0$ by

$$
\gamma \equiv \frac{1}{D^{\prime}\left(-E^{2}\right)^{1 / 2}}>0
$$

Then the operator $T_{\mu \nu}$ has the following properties:

(1) $T_{\mu \nu} \in \mathbf{B}\left(M_{\alpha}\right)$ and $\widetilde{T_{\mu \nu} f}=T_{\mu \nu} \tilde{f}, f \in \mathbf{B}\left(M_{\alpha}\right), \alpha= \pm \frac{1}{2}, 0$.

If $f$ is rotation invariant, then so is $T_{\mu \nu} f$.

(2) $\quad\left(d_{\nu \alpha} \frac{Q}{\sqrt{\omega^{3}}}, \frac{1}{\sqrt{\omega}} T_{\mu \nu} f\right)=\frac{e}{m+\delta}\left(d_{\mu \alpha} \frac{\hat{\rho}}{\sqrt{\omega^{3}}}, \frac{f}{\sqrt{\omega}}\right)-\left(\frac{\sqrt{\omega} c \gamma^{2}}{\sqrt{\epsilon} E^{2}} F_{\mu \alpha}, \frac{f}{\sqrt{\omega}}\right)$,

where $\delta=\epsilon \frac{e^{2}}{c^{2}}\left\|\frac{\hat{\rho}}{\omega}\right\|^{2}$ and $f \in M_{-\frac{1}{2}}$.

(3) $\left[\omega^{2}, T_{\mu \nu}\right]=\epsilon \frac{e}{c^{2}}\left(d_{\mu \nu} \hat{\rho}, \cdot\right) Q$.

(4) $T_{\mu \nu} \hat{\rho}=\delta_{\mu \nu} \frac{m}{e} Q$.

(5) $T_{\mu \nu}^{*} d_{\nu \alpha} T_{\alpha \beta}=d_{\mu \beta}-\theta \gamma^{2}\left(F_{\alpha \beta}, \cdot\right) F_{\alpha \mu}, \quad F_{\alpha \beta}=\sqrt{\epsilon} \frac{e}{c} \frac{\hat{\rho}^{2} d_{\alpha \beta}}{E^{2}+\omega^{2}}$.

(6) $e_{\mu}^{r} T_{\mu \nu} d_{\nu \alpha} T_{\alpha \beta}^{*} e_{\beta}^{s}=\delta_{r s}$.

(7) $e_{\mu}^{r} T_{\mu \nu} T_{\nu \alpha}^{*} e_{\alpha}^{s}=\delta_{r s}$.

(8) $\bar{T}_{\mu \nu} f=\gamma T_{\mu \nu} f+(1-\gamma)\left(\delta_{\mu \nu} f-\left(\frac{d-1}{d} V_{d}\right)^{-1}\left[d_{\mu \nu} f\right]\right), f \in M_{0}$ where $\gamma=\frac{D_{\epsilon+}}{D_{\epsilon-}},[f]=\int_{S_{d-1}} f(|k| \Omega) d \Omega$ and $d \Omega$ is volume element on $S_{d-1}$.

(9) $e_{\mu}^{r} \bar{T}_{\mu \nu} T_{\nu \alpha}^{*} e_{\alpha}^{s} f=\delta_{r s} f-(1-\gamma)\left(\frac{d-1}{d} V_{d}\right)^{-1} e_{\mu}^{r}\left[e_{\mu}^{s} f\right], f \in M_{0}$

(10) Let $h$ is a rotation invariant function. Then,

$$
\begin{aligned}
& T_{\mu \nu}^{*} d_{\nu \alpha} h T_{\alpha \beta}=\bar{T}^{*}{ }_{\mu \nu} d_{\nu \alpha} h \bar{T}_{\alpha \beta} \text {, on } M_{0} \\
& \left(d_{\nu \alpha} \frac{Q}{\sqrt{\omega^{3}}}, h T_{\mu \nu} f\right)=\left(d_{\nu \alpha} \frac{\bar{Q}}{\sqrt{\omega^{3}}}, h \bar{T}_{\mu \nu} f\right), h T_{\mu \nu} f \in M_{0}
\end{aligned}
$$

(11) $e_{\mu}^{(r)} T_{\mu \nu} F_{\nu \alpha}=0 . \quad(m<0)$. 
(12) $\left(\hat{\rho}, F_{\mu \nu}\right)=-m \frac{c}{\sqrt{\epsilon e}} \delta_{\mu \nu} . \quad(m<0)$

(13) $T_{\mu \nu}^{*} d_{\nu \alpha} Q=\theta \frac{c}{\sqrt{\epsilon}} \gamma^{\dot{2}} F_{\mu \alpha}+\frac{e}{m+\delta} d_{\mu \alpha} \hat{\rho}$.

(14) $\left(F_{\mu \alpha}, F_{\alpha \nu}\right)=\delta_{\mu \nu} \frac{1}{\gamma^{2}} . \quad(m<0)$.

Proof: See Ref.14.

We define the Fourier transform of the fields $A_{\mu}(f)$ and $\Pi_{\mu}(f)$ by

$$
\hat{A}_{\mu}(f)=A_{\mu}(\hat{f}), \hat{\Pi}_{\mu}(f)=\Pi_{\mu}(\hat{f}) .
$$

We put

$$
\begin{aligned}
B^{(r)}(f, p)= & \frac{1}{\sqrt{2}}\left\{\widehat{A}_{\mu}\left(\frac{1}{\sqrt{\hbar}} T_{\mu \nu}^{*} e_{\nu}^{r} \sqrt{c \omega} f\right)+i \hat{\Pi}_{\mu}\left(\frac{1}{\sqrt{\hbar}} T_{\mu \nu}^{*} e_{\nu}^{r} \frac{f}{\sqrt{c \omega}}\right)\right. \\
& \left.+\hbar p_{\nu}\left(\frac{1}{\sqrt{\hbar}} \frac{Q e_{\nu}^{r}}{(c \omega)^{\frac{3}{2}}}, f\right)\right\} \\
= & b^{(r)}(f)+p_{\mu}\left(L_{\mu}^{(r)}, f\right), f \in M_{0} \\
B^{\dagger(r)}(f, p)= & \frac{1}{\sqrt{2}}\left\{\widehat{A}_{\mu}\left(\frac{1}{\sqrt{\hbar}} \bar{T}_{\mu \nu}^{*} \tilde{e}_{\nu}^{r} \sqrt{c \omega} \tilde{f}\right)-i \widehat{\Pi}_{\mu}\left(\frac{1}{\sqrt{\hbar}} \bar{T}_{\mu \nu}^{*} \tilde{e}_{\nu}^{r} \frac{\tilde{f}}{\sqrt{c \omega}}\right)\right. \\
& \left.+\hbar p_{\nu}\left(\frac{1}{\sqrt{\hbar}} \frac{\bar{Q} e_{\nu}^{r}}{(c \omega)^{\frac{3}{2}}}, f\right)\right\} \\
= & b^{\dagger(r)}(f)+p_{\mu}\left(L_{\mu}^{(r)}, f\right), f \in M_{0},
\end{aligned}
$$

where

$$
L_{\mu}^{(r)}=\frac{\sqrt{\hbar} e_{\mu}^{r} Q}{\sqrt{2 c^{3} \omega^{3}}} \in M_{0} .
$$

By the definition of $A_{\mu}(f)$ and $\Pi_{\mu}(f)$, we can represent $b^{\sharp(r)}$ by linear combinations of $a^{\sharp(s)}$ 's:

$$
\begin{aligned}
& b^{(r)}(f)=a^{\dagger(s)}\left(W_{-}^{(r, s)} f\right)+a^{(s)}\left(W_{+}^{(r, s)} f\right), \\
& b^{\dagger(r)}(f)=a^{\dagger(s)}\left(\bar{W}_{+}^{(r, s)} f\right)+a^{(s)}\left(\bar{W}_{-}^{(r, s)} f\right),
\end{aligned}
$$

where $W_{ \pm}^{(r, s)}(1 \leq r, s \leq d-1)$ is operator definded by

$$
\begin{aligned}
& W_{+}^{(r, s)} f=\frac{1}{2}\left(\frac{1}{\sqrt{\omega}} e_{\mu}^{r} T_{\mu \nu}^{*} e_{\nu}^{s} \sqrt{\omega}+\sqrt{\omega} e_{\mu}^{r} T_{\mu \nu}^{*} e_{\nu}^{s} \frac{1}{\sqrt{\omega}}\right) f \\
& W_{-}^{(r, s)} f=\frac{1}{2}\left(\frac{1}{\sqrt{\omega}} e_{\mu}^{r} T_{\mu \nu}^{*} \tilde{e}_{\nu}^{s} \sqrt{\omega}-\sqrt{\omega} e_{\mu}^{r} T_{\mu \nu}^{*} \tilde{e}_{\nu}^{s} \frac{1}{\sqrt{\omega}}\right) \tilde{f} .
\end{aligned}
$$


Let

$$
\mathrm{W}_{ \pm}=\left(W_{ \pm}^{(r, s)}\right)_{1 \leq r, s \leq d-1}
$$

and

$$
\mathrm{L}=\left(L_{\mu}^{(r)}\right)_{1 \leq \mu \leq d, 1 \leq r \leq d-1}=\left(\begin{array}{lll}
L_{1}^{(1)} & \cdots & L_{d}^{(1)} \\
\vdots & & \vdots \\
L_{1}^{(d-1)} & \cdots & L_{d}^{(d-1)}
\end{array}\right)
$$

We see $\mathrm{W}_{ \pm} \in \mathrm{B}(\mathcal{W})$. For the vector of the form $\mathrm{f}=f_{1} \oplus \ldots \oplus f_{d-1} \in \mathcal{W}$, we define $B^{\sharp}(f, p)$ by

$$
\begin{aligned}
& B(\mathbf{f}, p) \equiv \sum_{r=1}^{d-1} B^{(r)}\left(f_{r}, p\right)=a^{\dagger}\left(\mathbf{W}_{-} \mathbf{f}\right)+a\left(\mathbf{W}_{+} \mathbf{f}\right)+(\mathbf{L} p, \mathbf{f}) \\
& B^{\dagger}(\mathbf{f}, p) \equiv \sum_{r=1}^{d-1} B^{\dagger(r)}\left(f_{r}, p\right)=a^{\dagger}\left(\overline{\mathbf{W}}_{+} \mathbf{f}\right)+a\left(\overline{\mathbf{W}}_{-} \mathbf{f}\right)+\left(\overline{\mathbf{L}}_{p}, \mathbf{f}\right)
\end{aligned}
$$

By virtue of Lemma $5.4(5)$ and (6), one can easily see that $W_{ \pm}$satisfy the following algebraic relations:

$$
\begin{aligned}
& \mathrm{W}_{+}^{*} \mathrm{~W}_{+}-\mathrm{W}_{-}^{*} \mathrm{~W}_{-}=I, \\
& \overline{\mathrm{W}}_{+}^{*} \mathrm{~W}_{-}-\overline{\mathrm{W}}_{-}^{*} \mathrm{~W}_{+}=0 \text {, } \\
& \mathrm{W}_{+} \mathrm{W}_{+}^{*}-\overline{\mathrm{W}_{-}} \overline{\mathrm{W}}_{-}^{*}=I \text {, } \\
& \mathrm{W}_{-} \mathrm{W}_{+}^{*}-\overline{\mathrm{W}}_{+} \overline{\mathrm{W}}_{-}^{*}=0 \text {. }
\end{aligned}
$$

We have seen in Section III that the above ralations are equivalent to that $\Xi(W) \in \Sigma$, where $\Sigma$ is the sympletic group over Hilbert space $\mathcal{W}$ and $\Xi(\mathrm{W})$ is defined by

$$
\Xi(\mathrm{W})=\left(\begin{array}{ll}
\mathrm{w}_{+} & \mathrm{W}_{-} \\
\bar{W}_{-} & \overline{\mathrm{W}}_{+}
\end{array}\right) .
$$

Lemma $5.5:$ We put $\mathcal{W}_{\alpha}=\underbrace{M_{\alpha} \oplus \ldots \oplus M_{\alpha}}_{d-1}, \alpha \in \mathbb{R}$. Then for $\mathbf{f} \in \mathcal{W}_{0} \cap \mathcal{W}_{1}$, we have

$$
\left[H_{\epsilon}(p), B^{\sharp}(\mathbf{f}, p)\right]= \pm B^{\sharp}(\hbar c \widehat{\omega} \mathbf{f}, p) \text {, on } \mathcal{F}_{0}^{E M} \cap D\left(H^{E M \frac{3}{2}}\right)
$$

where + (resp.-) corresponds to $B^{\dagger}$ (resp. B).

Proof: This follows directly from using Lemma 5.4 (3) and the commutation relations of $A_{\mu}, \Pi_{\mu}$ and $H^{E M}$. 
Now we can construct the Heisenberg field. For all $t \in \mathbb{R}$, we define the operators in $\mathcal{F}^{E M}$ as

$$
\begin{aligned}
A_{\mu}(f, t \mid p)= & \frac{1}{\sqrt{2}} \sum_{r=1}^{d-1}\left\{B^{\dagger(r)}\left(\frac{\sqrt{\hbar}}{\sqrt{c \omega}} e^{i c \omega t} e_{\nu}^{r} \bar{T}_{\mu \nu} \hat{f}, p\right)+B^{(r)}\left(\frac{\sqrt{\hbar}}{\sqrt{c \omega}} e^{-i c \omega t} e_{\nu}^{r} T_{\mu \nu} \tilde{\hat{f}}, p\right)\right\} \\
& -\frac{e}{m+\delta} \hbar p_{\nu}\left(d_{\mu \nu} \frac{\hat{\rho}}{\sqrt{(c \omega)^{3}}}, \frac{\hat{f}}{\sqrt{c \omega}}\right), \hat{f} \in M_{-\frac{1}{2}}, \\
\Pi_{\mu}(f, t \mid p)= & \frac{i}{\sqrt{2}} \sum_{r=1}^{d-1}\left\{B^{\dagger(r)}\left(\sqrt{c \omega} \sqrt{\hbar} e^{i c \omega t} e_{\nu}^{r} \bar{T}_{\mu \nu} \hat{f}, p\right)-B^{(r)}\left(\sqrt{c \omega} \sqrt{\hbar} e^{-i c \omega t} e_{\nu}^{r} T_{\mu \nu} \tilde{\hat{f}}, p\right)\right\}, \\
& \hat{f} \in M_{\frac{1}{2}} .
\end{aligned}
$$

Theorem $5.6:$ The operator $A_{\mu}(f, t \mid p),\left(\hat{f} \in M_{-\frac{1}{2}} \cap M_{\frac{1}{2}}\right)$ and $\Pi_{\mu}(f, t \mid p),\left(\hat{f} \in M_{\frac{1}{2}} \cap M_{\frac{3}{2}}\right)$ satisfy the Heisenberg equations Eqs.(5.2) and (5.3), respectively, on $\mathcal{F}_{0}^{E M} \cap D\left(H^{E M \frac{3}{2}}\right)$ with initial conditions $A_{\mu}(f, 0 \mid p)=A_{\mu}(f)$ and $\Pi_{\mu}(f, 0 \mid p)=\Pi_{\mu}(f)$, respectively. Furthermore if $\mathrm{f}$ is real, then for each $t \in \mathbb{R}, A_{\mu}(f, t \mid p)$ and $\Pi_{\mu}(f, t \mid p)$ are essentially self-adjoint on $\mathcal{F}_{0}^{E M}$.

Proof: By the definition of $B^{\sharp}$, we see that

$$
\begin{aligned}
A_{\mu}(f, 0 \mid p)= & \frac{1}{2}\left\{\hat{A}_{\alpha}\left(\bar{T}_{\alpha \beta}^{*} d_{\beta \nu} \bar{T}_{\mu \nu} \overline{\hat{f}}\right)+\hat{A}_{\alpha}\left(T_{\alpha \beta}^{*} d_{\beta \nu} T_{\mu \nu} \hat{f}\right)\right\} \\
& +\frac{1}{2} \hbar p_{\alpha}\left\{\left(\frac{\bar{Q} e_{\alpha}^{\tau}}{(c \omega)^{\frac{3}{2}}}, \frac{1}{\sqrt{c \omega}} e_{\nu}^{r} \bar{T}_{\mu \nu} \tilde{\hat{f}}\right)+\left(\frac{Q e_{\alpha}^{\tau}}{(c \omega)^{\frac{3}{2}}}, \frac{1}{\sqrt{c \omega}} e_{\nu}^{r} T_{\mu \nu} \tilde{\hat{f}}\right)\right\} .
\end{aligned}
$$

Hence the initial conditions are checked by Lemma $5.4(2),(3),(4),(5),(6)$ and (10). Using Lemma 5.5 , one can easily see that $A_{\mu}(f, t)$ and $\Pi_{\mu}(f, t)$ satisfy the Heisenberg equation Eq.(5.2) on $\mathcal{F}_{0}(\mathcal{H}) \cap$ $D\left(H^{E M \frac{3}{2}}\right)$, respectively. If $f$ is real, then $A_{\mu}(f, t \mid p)$ is symmetric operator. By the definition of $A_{\mu}(f, t \mid p)$, we see that for $\Phi \in D\left(\mathrm{~N}^{\frac{1}{2}}\right)$,

$$
\left\|A_{\mu}(f, t \mid p) \Phi\right\| \leq\left(2 M_{\mu}\right)\left\|(\mathrm{N}+1)^{\frac{1}{2}} \Phi\right\|+M_{\mu}(p)\|\Phi\|,
$$

where $M_{\mu}$ and $M_{\mu}(p)$ is independent of $t \in \mathbb{R}$. Hence one can easily see that for any $s \in \mathbb{R}$, the limit

$$
\lim _{N \rightarrow \infty} \sum_{n=0}^{N} \frac{\left\|A_{\mu}(f, t \mid p)^{n} \Psi\right\| s^{n}}{n !}<\infty, \Psi \in \mathcal{F}_{0}^{E M}(\mathcal{H})
$$

exsists uniformly in $t$. Hence $A_{\mu}(f, t \mid p)$ is essentially self adjoint on $\mathcal{F}_{0}^{E M}(\mathcal{H})$. The proof of the statements about $\Pi_{\mu}(f, t \mid p)$ is similar to that of $A_{\mu}(f, t \mid p)$.

For real fuction $f$, we denote the self-adjoint extentions of $A_{\mu}(f, t \mid p) \Gamma_{\mathcal{F}_{0}(\mathcal{H})}$ and $\Pi_{\mu}(f, t \mid p) \Gamma_{\mathcal{F}_{0}(\mathcal{H})}$ by the same symbols respectively. 
Lemma 5.7 : The operators $A_{\mu}(f, t \mid p)$ and $\Pi_{\mu}(f, t \mid p)$ satisfy the following equations:

$$
\begin{aligned}
& \text { (1) } A_{\mu}(f, t \mid p)=\exp \left(i \frac{t}{\hbar} H_{\epsilon}(p)\right) A_{\mu}(f) \exp \left(-i \frac{t}{\hbar} H_{\epsilon}(p)\right), \hat{f} \in M_{-\frac{1}{2}}, \\
& \text { (2) } \Pi_{\mu}(f, t \mid p)=\exp \left(i \frac{t}{\hbar} H_{\epsilon}(p)\right) \Pi_{\mu}(f) \exp \left(-i \frac{t}{\hbar} H_{\epsilon}(p)\right), \hat{f} \in M_{\frac{1}{2}} .
\end{aligned}
$$

Proof: Let us prove (1). Let $p \in \mathbb{R}^{d}$ fix throughout the proof. It suffices to prove the case where $f$ is real. For $\hat{f} \in M_{-\frac{1}{2}} \cap M_{\frac{1}{2}}$, we define

$$
\phi_{\mu}(t)=\exp \left(i A_{\mu}(f, t \mid p)\right), \mu=1, \ldots, d .
$$

Let $C^{\infty}=\bigcap_{n=0}^{\infty} D\left(\left(H^{E M}\right)^{n}\right)$. For $\Phi, \Psi \in \mathcal{F}_{0}^{E M} \cap C^{\infty}$, we can define

$$
\begin{aligned}
& F_{\mu}(t)=\left(\phi_{\mu}(t) \Phi, \Psi\right), \\
& F_{\mu}(t, n)=\left(\left(i A_{\mu}(f, t \mid p)\right)^{n} \Phi, \Psi\right) .
\end{aligned}
$$

Since $C^{\infty} \cap \mathcal{F}_{0}^{E M}$ is a set of analytic vectors of $A_{\mu}(f, t)$, we can see that

$$
F_{\mu}(t)=\lim _{N \rightarrow \infty} \sum_{n=0}^{N} \frac{1}{n !} F_{\mu}(t, n) .
$$

It follows from Lemma 5.6 that $F_{\mu}(t, n)$ is differentiable in $t$ with

$$
\frac{d}{d t} F_{\mu}(t, n)=\frac{-i}{\hbar}(-i)^{n}\left\{\left(A_{\mu}(f, t \mid p)^{n} \Phi, H_{\epsilon}(p) \Psi\right)-\left(H_{\epsilon}(p) \Phi, A_{\mu}(f, t \mid p)^{n} \Psi\right)\right\}
$$

Moreover, we can show

$$
\hbar \sum_{n=0}^{N} \frac{1}{n !}\left|\frac{d}{d t} F_{\mu}(t, n)\right| \leq \sum_{n=0}^{N} \frac{\left\|A_{\mu}(f, t \mid p)^{n} \Psi\right\|+\left\|A_{\mu}(f, t \mid p)^{n} \Phi\right\|}{n !} \times \text { const. }
$$

By the proof of Lemma 5.6, the r.h.s. of Eq.(5.7) converges uniformly in $t$ as $N \rightarrow \infty$. Then we have that $F_{\mu}(t)$ is also differentiable in $t$ with

$$
\begin{aligned}
\frac{d}{d t} F_{\mu}(t) & =\sum_{n=0}^{\infty} \frac{d}{d t} F_{\mu}(t, n) \\
& =\frac{-i}{\hbar}\left\{\left(\phi_{\mu}(t) \Phi, H_{\epsilon}(p) \Psi\right)-\left(H_{\epsilon}(p) \Phi, \phi_{\mu}(-t) \Psi\right)\right\} .
\end{aligned}
$$

Recall that $\mathcal{F}_{0}^{E M} \cap C^{\infty}$ is a core of $H_{\epsilon}(p)$. Hence, for $\Phi, \Psi \in D\left(H_{\epsilon}(p)\right)$, we can take $\Phi_{n}, \Psi_{n} \in$ $\mathcal{F}_{0}^{E M} \cap C^{\infty}$ such that $\Phi_{n} \rightarrow \Phi$ and $\Psi_{n} \rightarrow \Psi$ and $H_{\epsilon}(p) \Phi_{n} \rightarrow H_{\epsilon}(p) \Phi$ and $H_{\epsilon}(p) \Psi_{n} \rightarrow H_{\epsilon}(p) \Psi$, as 
$n \rightarrow \infty$. Then one can easily see that $\left(\phi_{\mu}(t) \Phi_{n}, H_{\epsilon}(p) \Psi_{n}\right)$ converges to $\left(\phi_{\mu}(t) \Phi, H_{\epsilon}(p) \Psi\right)$ uniformly in $t$, and $\left(\phi_{\mu}(t) \Phi_{n}, \Psi_{n}\right)$ to $\left(\phi_{\mu}(t) \Phi, \Psi\right)$. Hence we see that $\left(\phi_{\mu}(t) \Phi, \Psi\right)$ is also differentiable in $t$ with

$$
\frac{d}{d t}\left(\phi_{\mu}(t) \Phi, \Psi\right)=\left(\frac{i}{\hbar} \phi_{\mu}(t) \Phi, H_{\epsilon}(p) \Psi\right)-\left(\frac{i}{\hbar} H_{\epsilon}(p) \Phi, \phi_{\mu}(t) \Psi\right)
$$

Let $\theta(t)=\exp \left(i \frac{t}{\hbar} H_{\epsilon}(p)\right)$. We put

$$
G_{\mu}(t)=\left(\theta(-t) \phi_{\mu}(t) \theta(t) \Phi, \Psi\right), \quad \Phi, \Psi \in D\left(H_{\epsilon}(p)\right)
$$

Then we have that

$$
\begin{aligned}
& \frac{G_{\mu}(t+h)-G_{\mu}(t)}{h}=\left(\phi_{\mu}(t+h) \theta(t+h) \Phi, \frac{\theta(t+h)-\theta(t)}{h} \Psi\right) \\
& +\left(\theta(t+h) \Phi, \frac{\phi_{\mu}(-t-h)-\phi_{\mu}(-t)}{h} \theta(t) \Psi\right)+\left(\frac{\theta(t+h)-\theta(t)}{h} \Phi, \phi_{\mu}(-t) \theta(t) \Psi\right) \\
& \equiv I(h)+I I(h)+I I I(h) .
\end{aligned}
$$

One can easily see by Stone's theorem and the strong continuity of $\phi_{\mu}(t)$ in $t$ that

$$
\lim _{h \rightarrow 0}\{I(h)+I I I(h)\}=\frac{i}{\hbar}\left\{\left(\theta(-t) \phi_{\mu}(t) \theta(t) \Phi, H_{\epsilon}(p) \Psi\right)-\left(H_{\epsilon}(p) \Phi, \theta(-t) \phi_{\mu}(t) \theta(t) \Psi\right)\right\} .
$$

From Eq.(5.8) it follows that

$$
\lim _{h \rightarrow 0} I I(h)=\frac{-i}{\hbar}\left\{\left(\theta(-t) \phi_{\mu}(t) \theta(t) \Phi, H_{\epsilon}(p) \Psi\right)-\left(H_{\epsilon}(p) \Phi, \theta(-t) \phi_{\mu}(t) \theta(t) \Psi\right)\right\} .
$$

Thus $G_{\mu}(t)$ is differentiable in $t$ with

$$
\frac{d}{d t} G_{\mu}(t)=0
$$

Hence it follows from the initial condition that

$$
G_{\mu}(t)=\left(\exp \left(i A_{\mu}(f)\right) \Phi, \Psi\right)
$$

By Eq.(5.9), for all $s \in \mathbb{R}$, we have

$$
\exp \left(i s A_{\mu}(f, t \mid p)\right)=\theta(t) \exp \left(i s A_{\mu}(f)\right) \theta(-t)
$$

Each side of Eq.(5.10) is one parameter unitary group in $s$. Hence we get the desired result by Stone's theorem. By a limiting argument employing (2.1), we can extend (1) to any $f \in M_{-\frac{1}{2}}$. The proof of (2) is similar to that of (1). 


\subsection{A unitary transformation of $H_{\epsilon}$}

We denote the set of Hilbert-Schmidt operators on $\mathcal{W}$ by $I_{2}(\mathcal{W})$. Since $W_{-}^{(r, s)}$ has the integral kernel

$$
W_{-}^{(r, s)}\left(k, k^{\prime}\right)=\epsilon \frac{e}{c^{2}} \frac{e_{\mu}^{(r)}(k) e_{\mu}^{(s)}\left(k^{\prime}\right) \hat{\rho}(k) \bar{Q}\left(k^{\prime}\right)}{2\left(|k|+\left|k^{\prime}\right|\right)\left(|k|\left|k^{\prime}\right|\right)^{\frac{1}{2}}}
$$

one can easily see that $W_{-}^{(r, s)} \in L^{2}\left(\mathbb{P}^{d} \times \mathbb{R}^{d}\right)$. Hence we have

$$
W_{-} \in I_{2}(\mathcal{W})
$$

Lemma $5.8:$ For every $p \in \mathbb{R}^{d}$, there exists a unitary operator $\mathcal{U}(p)$ on $\mathcal{F}^{E M}$ such that

$$
\mathcal{U}^{-1}(p) B^{\sharp}(\mathrm{f}, p) \mathcal{U}(p)=a^{\sharp}(\mathrm{f}), \quad \mathrm{f} \in \mathcal{W}
$$

Proof: Note that $B^{\sharp}(\mathbf{f}, p)$ is just of the form of the operator $B^{\sharp}(f)$ considered in Theorem 3.4 with the folowing identifications:

$$
\mathcal{H}=\mathcal{W}, T=\mathrm{W}_{-}, S=\mathrm{W}_{+}, L=\mathrm{L} p
$$

Hence we obtain Eq.(5.11) by Theorem 3.4.

Lemma 5.9 : Let $\mathcal{U}(p) \Omega=\Omega(p)$. Then for all $p \in \mathbb{R}^{d}$,

$$
\Omega(p) \in D\left(H_{\epsilon}(p)\right)
$$

Proof: We set $\mathrm{K}=\mathrm{W}_{-} \mathrm{W}_{+}^{-1}$, and $\mathrm{H}=\sqrt{\omega} \mathrm{I}$. Since $\left\|\sqrt{\omega} W_{-}^{(r, s)}\right\|_{L^{2}\left(\mathbb{R}^{d} \times \mathbb{R}^{d}\right)}<\infty$, we have $\mathrm{HW}-\epsilon$ $I_{2}(\mathcal{W})$. It follows from the fact $\mathrm{H}^{T}\left(\mathrm{~K}+\mathrm{K}^{T}\right)^{*}=2 \overline{\mathrm{HK}}$ that $\mathrm{H}^{T}\left(\mathrm{~K}+\mathrm{K}^{T}\right)^{*} \in I_{2}(\mathcal{W})$. Then by Proposition 2.4, we obtain that $\Omega(p) \in D\left(H^{E M}\right)$. Since $D\left(H^{E M}\right)=D\left(H_{\epsilon}(p)\right), p \in \mathbb{R}^{d}$. we get the desired result.

Lemma 5.10 : For all $p \in \mathbb{R}^{d}, \mathbf{f} \in \mathcal{W}$,

$$
\exp \left(i \frac{t}{\hbar} H_{\epsilon}(p)\right) B(\mathrm{f}, p) \exp \left(-i \frac{t}{\hbar} H_{\epsilon}(p)\right)=B\left(e^{-i \hat{c} \hat{\omega}} f, p\right) .
$$


Proof: By the defenition of $B^{(r)}(f, p)$, Lemma $5.4(7),(9)$, and Lemma 5.8, one can see $\left(\widetilde{B}^{\dagger}(f)=\right.$ $\left.B^{\dagger}(\tilde{f})\right)$

$$
\begin{aligned}
& \exp \left(i \frac{t}{\hbar} H_{\epsilon}(p)\right) B^{(r)}(f, p) \exp \left(-i \frac{t}{\hbar} H_{\epsilon}(p)\right) \\
&=\frac{1}{\sqrt{2}}\left\{\widehat{A}_{\mu}\left(\frac{1}{\sqrt{\hbar}} T_{\mu \nu}^{*} e_{\nu}^{r} \sqrt{c \omega} f, t \mid p\right)+i \widehat{\Pi}_{\mu}\left(\frac{1}{\sqrt{\hbar}} T_{\mu \nu}^{*} e_{\nu}^{r} \frac{f}{\sqrt{c \omega}}, t \mid p\right)\right\}+p_{\nu}\left(L_{\nu}^{r}, f\right) \\
&=\frac{1}{2} B^{(s)}\left(e^{-i c \omega t} \frac{1}{\sqrt{\omega}} e_{\nu}^{s} T_{\nu \mu} T_{\mu \alpha}^{*} e_{\alpha}^{r} \sqrt{\omega} f+e^{-i c \omega t} \sqrt{\omega} e_{\nu}^{s} T_{\nu \mu} T_{\mu \alpha}^{*} e_{\alpha}^{r} \frac{f}{\sqrt{\omega}}\right) \\
&+\frac{1}{2} \widetilde{B}^{\dagger(s)}\left(e^{i c \omega t} \sqrt{\omega} e_{\nu}^{s} \bar{T}_{\nu \mu} T_{\mu \alpha}^{*} e_{\alpha}^{r} \frac{f}{\sqrt{\omega}}-e^{i c \omega t} \frac{1}{\sqrt{\omega}} e_{\nu}^{s} \bar{T}_{\nu \mu} T_{\mu \alpha}^{*} e_{\alpha}^{\tau} \sqrt{\omega} f\right) \\
&= B^{(r)}\left(e^{-i c \omega t} f, p\right), f \in M_{0} .
\end{aligned}
$$

Thus we get the desired result.

We set for $p \in \mathbb{R}^{d}$

$$
\mathcal{F}(p)=\mathcal{L}\left\{B^{\dagger}\left(f_{1}, p\right), \ldots, B^{\dagger}\left(f_{n}, p\right) \Omega(p), \Omega(p) \mid f_{j} \in \mathcal{W}, j=1, \ldots, n, n \geq 1\right\}
$$

As was seen in Eqs.(3.7) and (3.8) in the abstract case, $a^{\sharp}(f)$ can be represented by $B^{\sharp}(f)$. Hence $\mathcal{F}(p)$ is irreducible in $a^{l}$ in the sense of Berezin. This implies that $\mathcal{F}(p)$ is dense in $\mathcal{F}^{E M}$. Lemmas 5.9 and 5.10 gives the following corollary.

Corollary 5.11 : For all $p \in \mathbb{R}^{d}$, there exists a real number $E(p)$ such that

$$
\exp \left(i \frac{t}{\hbar} H_{\epsilon}(p)\right) \Omega(p)=\exp \left(i \frac{t}{\hbar} E(p)\right) \Omega(p)
$$

Proof: Using the denseness of $\mathcal{F}(p)$, we can easily see that if there exists $\Phi \in \mathcal{F}^{E M}$ such that

$$
B(\mathrm{f}, p) \Phi=0, \text { for all } \mathrm{f} \in \mathcal{W}
$$

then $\Phi$ is a constant multiple of $\Omega(p)$. On the other hand, from Lemma 5.10 it follows that for $p \in \mathbb{R}^{d}$

$$
B(\mathrm{f}, p) \exp \left(i \frac{t}{\hbar} H_{\epsilon}(p)\right) \Omega(p)=0, \text { for all } \mathbf{f} \in \mathcal{W}
$$

It follows from Lemma 5.9 that $\left.\exp i(t / \hbar) H_{\epsilon}(p)\right) \Omega(p)$ has to be of the form Eq.(5.12).

Corollary 5.11 means that $E(p)$ is an eigenvalue of $H_{\epsilon}(p)$. 
Lemma 5.12 : The eigenvalue $E(p)$ is explicitly given by

$$
E(p)=\frac{1}{2 m} \hbar^{2} p^{2}\left(1-2 \Lambda+\epsilon \Lambda^{2}\right)-\eta
$$

where

$$
\Lambda=\frac{e}{2 c^{2}}\left(\frac{Q e_{\mu}^{r}}{\sqrt{\omega^{3}}},\left(\mathrm{~W}_{+}^{-1}\right)^{(r, s)} \frac{\hat{\rho} e_{\mu}^{s}}{\sqrt{\omega}}\right)_{0}, \eta=\frac{\epsilon e^{2}}{8 m c}\left(\frac{\hat{\rho} e_{\mu}^{r}}{\sqrt{\omega}},\left(\mathrm{W}_{-} \mathrm{W}_{+}^{-1}\right)^{(r, s)} \frac{\hat{\rho} e_{\mu}^{s}}{\sqrt{\omega}}\right)_{0} .
$$

Proof: See Appendix A.

We define $m^{\prime}, m_{0}$ and $m_{00}$ by

$$
\begin{aligned}
\frac{1}{2 m^{\prime}} & =\frac{1}{2 m}-\frac{2 \Lambda}{2 m}+\frac{\epsilon \Lambda^{2}}{2 m} \\
\frac{1}{m_{0}} & =\frac{2 \Lambda}{m} \\
\frac{1}{m_{00}} & =\frac{\epsilon \Lambda^{2}}{m} .
\end{aligned}
$$

Lemma $5.13:$ For $p \in \mathbb{R}^{d}$, the unitary operator $\mathcal{U}(p)$ maps $D\left(H^{E M}\right)$ onto itself with

$$
\mathcal{U}^{-1}(p) H_{\epsilon}(p) \mathcal{U}(p)=H^{E M}+E(p), \text { on } D\left(H^{E M}\right)
$$

Proof: Making use of Lemma 5.10 and Corolllary 5.11, one can see that on $\mathcal{F}(p)$,

$$
\exp \left(i \frac{t}{\hbar} H_{\epsilon}(p)\right)=\mathcal{U}(p) \exp \left\{i c t d \Gamma(\widehat{\omega})+i \frac{t}{\hbar} E(p)\right\} \mathcal{U}^{-1}(p)
$$

Since each side of Eq.(5.13) is a one parameter unitary group in $t$, we get Lemma 5.13.

As was seen in Lemmas 3.2 and 3.3 and Theorem 3.4, the operator $\mathcal{U}(p)$ can be represented by the Intertwining operator $\mathrm{U}$ and the Displacement operator $\mathrm{S}(p)$ :

$$
\mathcal{U}(p)=\mathbf{S}(p) \mathbf{U}
$$

By Lemma 3.3, one can see that $\mathrm{S}(p)$ has the form " $\exp \left(-i p_{\mu} \pi\left(L_{\mu}\right)\right)$ ", where

$$
p_{\mu} \pi\left(L_{\mu}\right)=i \frac{e}{m+\delta} p_{\mu} \sum_{r=1}^{d-1}\left\{a^{(r)}\left(\frac{e_{\mu}^{r} \hat{\rho}}{\sqrt{2 \hbar c^{3} \omega^{3}}}\right)-a^{\dagger(r)}\left(\frac{e_{\mu}^{r} \hat{\rho}}{\sqrt{2 \hbar c^{3} \omega^{3}}}\right)\right\} .
$$

It is well known that $\mathbf{S}(p)$ maps from $D\left(H^{E M}\right)$ onto itself. Then, from the proof of Lemma 5.13 it also follows that $U$ maps from $D\left(H^{E M}\right)$ onto itself. We define unitary operators on $\mathcal{F}$ by

$$
\widehat{\mathrm{U}}=\mathrm{F}^{-1}\left(\int_{\mathbb{R}^{d}}^{\oplus} \mathrm{U} d p\right) \mathbf{F}, \quad \widehat{\mathbf{S}}=\mathrm{F}^{-1}\left(\int_{\mathbb{\boxplus}^{d}}^{\oplus} \mathrm{S}(p) d p\right) \mathbf{F}, \quad \hat{U}=\widehat{\mathrm{S}} \widehat{\mathrm{U}} .
$$


Put

$$
T=i \frac{e}{m+\delta} \mathrm{p}_{\mu} \otimes \sum_{r=1}^{d-1}\left\{a^{(r)}\left(\frac{e_{\mu}^{\tau} \hat{\rho}}{\sqrt{2 \hbar c^{3} \omega^{3}}}\right)-a^{\dagger(r)}\left(\frac{e_{\mu}^{\tau} \hat{\rho}}{\sqrt{2 \hbar c^{3} \omega^{3}}}\right)\right\}
$$

Then, by Eq.(5.15), one can easily see that $\widehat{\mathrm{S}}=\exp (-i T)$ and $\widehat{\mathrm{U}}=I \otimes \mathrm{U}$. It is well known that $\widehat{\mathrm{S}}$ maps $D_{0} \equiv D\left(\mathrm{p}^{2} \otimes I\right) \cap D\left(I \otimes H^{E M}\right)$ onto itself (Ref.1). Then we see that $\hat{\mathrm{U}}$ maps $D_{0}$ onto itself.

Theorem 5.14 : The unitary operator $\hat{\mathcal{U}}$ maps $D_{0}$ onto itself and satisfies

$$
\hat{\mathcal{U}}^{-1} H_{\epsilon} \hat{\mathcal{U}}=\frac{1}{2 m^{\prime}} \mathrm{p}^{2} \otimes I+I \otimes H^{E M}-\eta \text {, on } D_{0}
$$

Proof: For $f, g \in D\left(\mathbf{p}^{2}\right)$ and $\Phi, \Psi \in D\left(H^{E M}\right)$,

$$
\begin{aligned}
-\left(\hat{\mathcal{U}}^{-1} H_{\epsilon} \hat{\mathcal{U}}(f \otimes \Phi), g \otimes \Psi\right) & =\int d p \overline{\hat{f}}(p) \hat{g}(p)\left(H_{\epsilon}(p) \mathrm{S}(p) \mathrm{U} \Phi, \mathrm{S}(p) \mathrm{U} \Psi\right) \\
& =\int d p \overline{\hat{f}}(p) \hat{g}(p)\left(\left(E(p)+H^{E M}\right) \Phi, \Psi\right) \\
& =\left(\left(\frac{1}{2 m^{\prime}} \mathrm{p}^{2} \otimes I+I \otimes H^{E M}-\eta\right)(f \otimes \Phi), g \otimes \Psi\right) .
\end{aligned}
$$

Then Eq.(5.16) follows on $D\left(\mathrm{p}^{2}\right) \hat{\otimes} D\left(H^{E M}\right)$. Since $D\left(\mathrm{p}^{2}\right) \hat{\otimes} D\left(H^{E M}\right)$ is a core of the r.h.s.of Eq.(5.16), Theorem 5.14 follows.

We note that the operator $b^{\sharp}$ and the Intertwinig operator $U$ are independent of $p \in \mathbb{R}^{d}$. Hence we can see that they are concerned with only the self interaction $A^{2}(\rho)$ of the quantized radiation field. In fact putting $\epsilon=0$, we get $\mathrm{W}_{+}=I, \mathrm{~W}_{-}=0$ so that in this case $\mathrm{U}=I, b^{\sharp}=a^{\sharp}$ holds. Moreover, on the Displacement operator $\widehat{\mathrm{S}}$, if $\epsilon>0$, the electron mass term " $m+\delta$ " appears in the generator $T$ (see Eq.(5.15)). This implies that " $\delta$ " comes from an effect of the self-interaction of quantized radiation field.

\section{AN ASYMPTOTIC BEHAVIOR OF THE TOTAL HAMIL- TONIAN}

In this section we shall discuss an asymptotic behavior of the total Hamiltonian of the coupled system of the one electron atom and the quantized radiation field with the dipole approximation.

We take the potential $V(x)$ of the atom to be a real valued measurable function on $\mathbb{R}^{d}$ which satisfies 
$(\mathrm{V}-1): D\left(\mathbf{p}^{2}\right) \subset D(V)$ and for all $\lambda>0, V\left(\mathbf{p}^{2}+\lambda\right)^{-1}$ is bounded with

$$
\lim _{\lambda \rightarrow \infty}\left\|V\left(\mathrm{p}^{2}+\lambda\right)^{-1}\right\|=0
$$

$(\mathrm{V}-2)$ : For all $t>0, x \in \mathbb{R}^{d}$

$$
\int_{\mathbb{R}^{d}} e^{-t|x-y|^{2}}|V(y)| d y<\infty
$$

Condition ( $\mathrm{V}-1$ ) implies that the Hamiltonian of the atom

$$
H_{A} \equiv \frac{\mathrm{p}^{2}}{2 m}+V
$$

is self-adjoint on $D\left(p^{2}\right)$ (Ref.1). Taking the mass renormalization into account, the mass-renormalized total Hamiltonian of our model is defined by

$$
H_{\epsilon}^{r e n}(V)=H_{A}^{r e n}(V) \otimes I+H_{I}^{(1)}+\epsilon H_{I}^{(2)}+I \otimes H^{E M}
$$

where

$$
H_{A}^{r e n}(V)=\frac{1}{2 m_{0}} \mathrm{p}^{2}+V, H_{I}^{(1)}=\frac{e}{m} \mathrm{p}_{\mu} \otimes A_{\mu}(\rho), H_{I}^{(2)}=\frac{e^{2}}{2 m} I \otimes A^{2}(\rho)
$$

For $\kappa>0$, we introduce

$$
c(\kappa)=c \kappa, \quad e(\kappa)=e \kappa^{-\frac{1}{2}}, \quad m(\kappa)=m \kappa^{-2},
$$

which are regarded as a scaled speed of light, a scaled elementary charge and a scaled mass of the electron respectively. We define the scaled Hamiltonian $H_{\epsilon}^{r e n}(V, \kappa)$ by replacing the parameters $c, e, m$ in $H_{\epsilon}^{\text {ren }}(V)$ by $c(\kappa), e(\kappa), m(\kappa)$ respectively:

$$
H_{\epsilon}^{r e n}(V, \kappa)=H_{A}^{r e n}(V, \kappa) \otimes I+\kappa H_{I}^{(1)}+\epsilon H_{I}^{(2)}+\kappa I \otimes H^{E M},
$$

where

$$
H_{A}^{r e n}(V, \kappa)=\frac{1}{2 m_{0}(\kappa)} \mathrm{p}^{2}+V
$$

$m_{0}(\kappa)$ is the scaled mass; $c, e, m$ in $m_{0}$ replaced by $c(\kappa), e(\kappa), m(\kappa)$, respectively. From now on, for any object $A$ (e.g., operators, vectors, etc.) which has parameters $c, e, m$, we denote by $A(\kappa)$ the object with $c, e, m$ replaced by $c(\kappa), e(\kappa), m(\kappa)$, respectively. 
We want to consider the scaling limit $\kappa \rightarrow \infty$ of $H_{\epsilon}^{r e n}(V, \kappa)$. Obviously $c(\kappa) \rightarrow \infty, e(\kappa) \rightarrow$ $0, m(\kappa) \rightarrow 0(\kappa \rightarrow \infty)$, in this sense, the scaling limit $\kappa \rightarrow \infty$ in $H_{\epsilon}(V, \kappa)$ coresponds to the nonrelativistic limit at the same time as the magnitude of the coupling charge and the mass of the electron become 0. In Ref.1, the scaling limit of the Pauli-Fierz model has been studied with a different scaling $c(\kappa)=c \kappa$ and $e(\kappa)=e \kappa^{\frac{3}{2}}$. In that case, the scaled Hamiltonian, $H_{P F}^{r e n}(V, \kappa)$, which is taken another mass renormalization into account, is given by

$$
H_{P F}^{r e n}(V, \kappa)=H_{P F, A}^{r e n}(V, \kappa) \otimes I+\kappa H_{I}^{(1)}+\kappa I \otimes H^{E M}
$$

where $H_{P F, A}^{r e n}$ denotes the renormalized Hamiltonian of the atom (Ref.1). Without the difference of mass renormalization from ours, Eq.(6.1) can be regarded as $H_{0}^{r e n}(V, \kappa)(\epsilon=0)$ in our model. In this sense our model $H_{\epsilon}^{r e n}(V, \kappa)$ is a generarized one. We set

$$
\begin{aligned}
& C_{\kappa}(V)=\hat{u}(\kappa)^{-1}(V \otimes I) \hat{u}(\kappa), 0<\kappa<\infty, \\
& C(V)=\hat{\mathcal{U}}^{-1}(V \otimes I) \hat{\mathcal{U}}
\end{aligned}
$$

and define $\widetilde{H}_{\epsilon}^{r e n}(V, \kappa)$ by

$$
\tilde{H}_{\epsilon}^{r e n}(V, \kappa)=\frac{1}{2 m_{00}(\kappa)} \mathrm{p}^{2} \otimes I+\kappa I \otimes H^{E M}-\eta(\kappa)+C_{\kappa}(V) .
$$

Lemma 6.1 : The following limits exist:

(1) $\lim _{\kappa \rightarrow \infty} \mathrm{W}_{+}(\kappa)=I$ in operator norm,

(2) $\lim _{\kappa \rightarrow \infty} \mathrm{W}_{-}^{*}(\kappa) \mathrm{W}_{-}(\kappa)=0$ in trace norm,

(3) $s-\lim _{\kappa \rightarrow \infty} L_{\mu}^{(r)}(\kappa)=\frac{e}{m} \frac{e_{\mu}^{r} \sqrt{\hbar}}{\sqrt{2 c^{3} \omega^{3}}} \hat{\rho} \equiv L_{\mu, \infty}^{(r)}$.

Proof: (1) Let us prove the convergence of $T_{\mu \nu}^{*}(\kappa)$ in $\kappa$ first. For any $f \in M_{-\frac{1}{2}}$, using Lemma 5.3 (3), we see that for sufficiently large $\kappa>0$

$$
\left\|\delta_{\mu \nu} f-T_{\mu \nu}^{*}(\kappa) f\right\|_{-\frac{1}{2}} \leq \epsilon \frac{e^{2}}{c^{2}} \frac{\left\|\hat{\rho} \omega^{d-\frac{5}{2}}\right\|_{\infty}\|\hat{\rho} \sqrt{\omega}\|_{\infty}\|G\|_{\left(1-\frac{d}{2}\right)}\|f\|_{-\frac{1}{2}}}{m \kappa-\left(m+d_{m}\right)} .
$$

Hence we have

$$
\lim _{\kappa \rightarrow \infty} T_{\mu \nu}^{*}(\kappa)=\delta_{\mu \nu} I \text { in opeator norm in } \mathrm{B}\left(M_{-\frac{1}{2}}\right)
$$


In the same way one can easily see that $T_{\mu \nu}^{*}(\kappa) \rightarrow \delta_{\mu \nu} I$ as $\kappa \rightarrow \infty$ in operator norm in $\mathbf{B}\left(M_{\frac{1}{2}}\right)$. Then from the definition of $W_{+},(1)$ follows. Since the trace norm of $W_{-}^{*}(\kappa) W_{-}(\kappa)$ is $\left\|W_{-}^{(r, s)}(\cdot, \cdot)\right\|_{L^{2}\left(\mathbb{R}^{d} \times \mathbb{R}^{d}\right)}^{2}$, we have

$$
\left\|W_{-}^{(\tau, s)}(\cdot, \cdot)\right\|_{L^{2}\left(\mathbb{R}^{d} \times \mathbb{B}^{d}\right)}^{2} \leq \frac{1}{4} \epsilon \frac{e^{2}}{c^{2}} \frac{\left\|\hat{\rho} / \sqrt{\omega^{3}}\right\|^{2}\|\hat{\rho} / \sqrt{\omega}\|^{2}}{\left(m \kappa-\left(m+d_{m}\right)\right)^{2}} \rightarrow 0,
$$

as $\kappa \rightarrow \infty$. Thus (2) follows. (3) is trivial.

Let $\mathbf{S}_{0}(p)$ be the Displacement operator corresponding to the case $\epsilon=0$ for $p \in \mathbb{R}^{d}$ and put

$$
\widehat{\mathbf{S}}_{0}=\int_{\mathbb{R}^{d}}^{\oplus} \mathbf{S}_{0}(p) d p .
$$

Lemma 6.2 : The strong limit of $\hat{\mathcal{U}}(\kappa)$ exists as $\kappa \rightarrow \infty$ with

$$
-\quad s-\lim _{\kappa \rightarrow \infty} \hat{U}(\kappa)=\widehat{\mathrm{S}}_{0} .
$$

Proof: For any $p \in \mathbb{R}^{d}$, note that $\mathcal{U}(p, \kappa)=\mathrm{S}(p, \kappa) \mathrm{U}(\kappa)$ and $\mathrm{S}_{0}(p)$ are just of the operators $\mathcal{U}(\kappa)=$ $\mathrm{S}(\kappa) \mathrm{U}(\kappa)$ and $\mathcal{U}=\mathrm{SU}$ considered in Theorem 3.12, respectively, with the following identifications:

$$
\begin{aligned}
& T(\kappa)=\mathrm{W}_{-}(\kappa), S(\kappa)=\mathrm{W}_{+}(\kappa), L(\kappa)=p_{\mu} L_{\mu}^{(r)}(\kappa), \\
& T=0, S=I, L=p_{\mu} L_{\mu, \infty}^{(r)} .
\end{aligned}
$$

By Lemma 6.1, the conditions $(A-1)-(A-4)$ in Section III are checked. Hence Theorem 3.12 yields

$$
s-\lim _{\kappa \rightarrow \infty} \mathcal{U}(p, \kappa)=\mathrm{S}_{0}(p) .
$$

For every $\Phi \in \mathcal{F}$, we put $(\mathrm{F} \Phi)(p)=\Phi(p)$, where $\mathbf{F}$ is the unitary operator defined in Section 5 . Then

$$
\left\|\widehat{\mathcal{U}}(\kappa) \Phi-\widehat{\mathrm{S}}_{0} \Phi\right\|^{2}=2\|\Phi\|^{2}-2 \operatorname{Re} \int_{\mathbb{R}^{d}}\left(\mathcal{U}(p, \kappa) \Phi(p), \mathrm{S}_{0}(p) \Phi(p)\right) d p
$$

The integrand in (6.4) can be estimated as

$$
\left|\left(\mathcal{U}(p, \kappa) \Phi(p), \mathbf{S}_{0} \Phi(p)\right)\right| \leq\|\Phi(p)\|^{2}
$$

The r.h.s of Eq.(6.5) is integrable. Hence the dominated convergense theorem and Eq.(6.3) yield

$$
\lim _{\kappa \rightarrow \infty} \int_{\mathbb{R}^{d}}\left(\mathcal{U}(p, \kappa) \Phi(p), \mathrm{S}_{0}(p) \Phi(p)\right) d p=\|\Phi\|^{2} .
$$


Thus Eq.(6.2) follows.

We shall next check the conditions $(C-1)-(C-3)$ in Section IV with the following identifcations:

$$
\begin{aligned}
& \mathcal{H}=L^{2}\left(\mathbb{R}^{d}\right), \mathcal{K}=\mathcal{W}, C_{\kappa}=C_{\kappa}(V), \\
& A=\mathrm{p}^{2}, B=H^{E M}, F_{1}(\kappa)=\frac{1}{2 m_{00}(\kappa)}, F_{2}(\kappa)=\eta(\kappa) .
\end{aligned}
$$

$(\mathbf{C}-1)^{\prime}:$ From the assumption $(\mathrm{V}-1)$ it follows that $\left(\mathbf{p}^{2}+\lambda\right)^{-1}$ and $\widehat{\mathcal{U}}(\kappa)$ are commute. Thus for all $\kappa>0, D\left(\mathrm{p}^{2} \otimes I\right) \subset D\left(C_{\kappa}(V)\right)$ and $C_{\kappa}(V)\left(\mathrm{p}^{2} \otimes I+\lambda\right)^{-1}$ is bounded operator for all $\lambda>0$ with

$$
\lim _{\kappa \rightarrow \infty}\left\|C_{\kappa}(V)\left(\mathrm{p}^{2} \otimes I+\lambda\right)^{-1}\right\|=0
$$

the convergence is uniform in $\kappa>0$.

$(\mathrm{C}-2)^{\prime}:$ By virtue of Lemma 6.2 , we see that

$$
s-\lim _{\kappa \rightarrow \infty} C_{\kappa}(V)\left(\mathrm{p}^{2} \otimes I+\lambda\right)^{-1}=C(V)\left(\mathrm{p}^{2} \otimes I+\lambda\right)^{-1} .
$$

$(\mathrm{C}-3)^{\prime}:$ It is easy to see that

$$
\begin{aligned}
& \text { (1) } s-\lim _{\kappa \rightarrow \infty} \frac{1}{2 m_{00}(\kappa)}=\frac{\epsilon}{2 m}\left(\frac{e^{2}}{2 m c^{2}}\right)^{2}\left\|\frac{\dot{\rho}}{\omega}\right\|^{4} \equiv \frac{1}{2 m_{\infty}} \\
& \text { (2) } s-\lim _{\kappa \rightarrow \infty} \eta(\kappa)=0 .
\end{aligned}
$$

By $(\mathbf{C}-1)^{\prime}-(\mathbf{C}-3)^{\prime}$ and Lemma 4.1 , it can be seen that $\tilde{H}_{\epsilon}^{r e n}(V, \kappa)$ is self-adjoint on $D_{0}$ for all $\kappa>0$, bounded from below and essentially self-adjoint on any core of

$$
\frac{1}{2 m_{00}(\kappa)} \mathrm{p}^{2} \otimes I+\kappa I \otimes H^{E M}-\eta(\kappa) .
$$

The fundamental theorem concerning our model $H_{\epsilon}^{r e n}(V, \kappa)$ is the following:

Theorem 6.3 : For all $\kappa>0$, the unitary operator $\hat{\mathcal{U}}(\kappa)$ maps $D_{0}$ onto itself and satisfies

$$
\widehat{\mathcal{U}}(\kappa)^{-1} H_{\epsilon}^{r e n}(V, \kappa) \hat{\mathcal{U}}(\kappa)=\tilde{H}_{\epsilon}^{r e n}(V, \kappa) \text {, on } D_{0}
$$

In particular, $H_{\epsilon}^{r e n}(V, \kappa)$ is self-adjoint on $D_{0}$ for all $\kappa>0$ and bounded from below. 
Proof: The unitary equivalence follows from Theorem 5.14, the self-adjointness from that of $\widetilde{H}_{\epsilon}^{r e n}(V, \kappa)$ on $D_{0}$.

Theorem 6.3 means that the effect of the interactions of the one electron atom and the quantized radiation field, and self-interaction of the field, $H_{I}^{(1)}$ and $H_{I}^{(2)}$, in $H_{\epsilon}^{r e n}(V, \kappa)$ can be regarded as the transformed potential $C_{\kappa}(V)$ in the decoupled Hamiltonian $\tilde{H}_{\epsilon}^{r e n}(V, \kappa)$. In Ref.1, $C_{\kappa}(V)$ formally can be regarded as the potential which has a fluctuation in the position of the electron.

Lemma 6.4 : The operators $C_{\kappa}(V), 0<\kappa<\infty$ and $C(V)$ are in $\mathbb{I}(\mathcal{F})$ with

$$
\begin{aligned}
& D_{L^{2}\left(\mathbb{R}^{d}\right)}\left(C_{\kappa}(V)\right)=D_{L^{2}\left(\mathbb{R}^{d}\right)}(C(V))=D\left(\mathrm{p}^{2}\right), \\
& D_{\mathcal{F}^{E M} M}\left(C_{\kappa}(V)\right)=D_{\mathcal{F}^{E M}}(C(V))=D\left(H^{E M}\right) .
\end{aligned}
$$

The partial expectations $E_{\Omega}\left(C_{\kappa}(V)\right)$ and $E_{\Omega}(C(V))$ of $C_{\kappa}(V)$ and $C(V)$, respectively, with respect to $\Omega$ are given by

$$
\begin{aligned}
& E_{\Omega}\left(C_{\kappa}(V)\right)=V_{e f f, \kappa}, \text { on } D\left(\mathbf{p}^{2}\right) \\
& E_{\Omega}(C(V))=V_{e f f}, \text { on } D\left(\mathbf{p}^{2}\right)
\end{aligned}
$$

where $V_{e f f, \kappa}$ and $V_{\text {eff }}$ are the multiplication operators associated with the functions

$$
\begin{aligned}
& V_{e f f, \kappa}(x)=\left(2 \pi C_{\kappa}(\rho)\right)^{-\frac{d}{2}} \int d y e^{-|x-y|^{2} / 2 C_{\kappa}(\rho)} V(y), 0<\kappa<\infty, \\
& V_{e f f}(x)=(2 \pi C(\rho))^{-\frac{d}{2}} \int d y e^{-|x-y|^{2} / 2 C(\rho)} V(y), \\
& C_{\kappa}(\rho)=\frac{d-1}{2 d}\left(\frac{\hbar}{m c}\right)^{2} \frac{e^{2}}{\hbar c} \int d k \frac{|\hat{\rho}(k)|^{2}}{\omega(k)^{3}\left(1-\Delta_{\kappa, \epsilon}(k)\right)}, 0<\kappa<\infty, \\
& C(\rho)=\frac{d-1}{2} \frac{1}{m c}\left(\frac{\hbar}{m c}\right)^{2} \frac{e^{2}}{\hbar c} \int d k \frac{|\hat{\rho}(k)|^{2}}{\omega(k)^{3}}, \\
& \text { where } \quad \Delta_{\kappa, \epsilon}(k)=\frac{1}{\kappa} \epsilon \frac{e^{2}}{m c^{2}} \frac{d-1}{d} \int \frac{\hat{\rho}^{2}\left(k^{\prime}\right)}{k^{2}-k^{\prime 2}+i 0} d k^{\prime} .
\end{aligned}
$$

Proof: The proof is same as that of Lemma 3.3 in Ref.1. Hence it is omited.

In Ref.1, it was shown that $C(\rho)$ can be interpreted as the mean squre fluctuation in the position of a free electron. We show the main theorem in the present paper.

Theorem 6.5 : For all $z \in \mathbb{C}$ with $\operatorname{Im} z \neq 0$ or $z<0$ with $|z|$ sufficiently large,

$$
s-\lim _{\kappa \rightarrow \infty}\left(H_{\epsilon}^{r e n}(V, \kappa)-z\right)^{-1}=\widehat{\mathrm{S}}_{0}\left\{\left(\frac{1}{2 m_{\infty}} \mathrm{p}^{2}+V_{\text {eff }}+z\right)^{-1} \otimes P_{0}\right\} \widehat{\mathrm{S}}_{0}^{-1},
$$


where $P_{0}$ is the projection from $\mathcal{F}^{E M}$ to $\{\alpha \Omega \mid \alpha \in \mathbb{C}\}$.

Proof: By the same identification as that of stated in $(\mathbf{C}-1)^{\prime}-(\mathbf{C}-3)^{\prime}$ and Lemmas 4.2, 6.2 and 6.3 , we can prove Theorem 6.5 .

\section{REMARKS}

(1) The operator $V_{\text {eff }}$ is called an "effective potential". The effective potential $V_{\text {eff }}$ coincides with that of Ref.1. However in the case $\kappa<\infty$, the partial expectation $V_{\text {eff }, \kappa}$ in terms of Fock vacuum dose not coincide with that of Ref.1.

(2) In Ref.1, scaled parameters $c(\kappa)$ and $e(\kappa)$ are introduced by

$$
c(\kappa)=c \kappa, \quad e(\kappa)=e \kappa^{\frac{3}{2}}
$$

respectively and $m$ is not scaled. In this scaling case, it follows from Theorem 6.4 that

$$
\begin{aligned}
\lim _{\kappa \rightarrow \infty} C_{\kappa}(\rho) & =0 \\
\lim _{\kappa \rightarrow \infty} V_{e f f, \kappa}(x) & =V(x), \quad x \in \mathbb{R}^{d} .
\end{aligned}
$$

Then we expect that the effective potential becomes trivial.

(3) We introduce a renormalized electron mass $m_{\text {ren }}$ by:

$$
m_{r e n}=m-\delta
$$

and the interaction Hamiltonian with the renormalized mass $m_{r e n}$ by:

$$
H^{m r e n}=\frac{1}{2 m_{r e n}}(\mathbf{p} \otimes I+e A(\rho))^{2}+I \otimes H^{E M} .
$$

Let $H^{m r e n}(\kappa)$ be the scaled interaction Hamiltonian for $H^{m r e n}$ with parameters Eq.(6.6). The generator of the Displcement operator for $H^{m r e n}(\kappa)$ (see Eq.(5.15)) is independent on $\kappa>0$ and coincides with $\widehat{\mathrm{S}}_{0}$ stated in Lemma 6.2. Since for sufficiently large $\kappa>0, m_{r e n}(\kappa)<0$, we see that $H^{m r e n}(\kappa)$ with sufficiently large $\kappa>0$ becomes the unphysical interaction Hamiltonian. In Appendix $B$, we show that in the case where the interaction Hamiltohian has a negative electron mass,

$$
\Xi(W) \notin \Sigma .
$$


Hence for $H^{m r e n}(\kappa)$ with sufficiently large $\kappa>0$, (6.7) holds. Then it implies that the unitary operator $\widehat{\mathcal{U}}(\kappa)$ satisfing Theorem 6.3 can not be constructed for $H^{m r e n}(\kappa)$ with sufficiently larg $\kappa>0$.

\section{ACKNOWLEDGMENTS}

The author would like to thank Professor A.Arai for helpful discussions. 


\section{APPENDIX A}

In this appendix, we prove Lemma 5.12. In order to do that, we shall prepare some lemmas. For $p \in \mathbb{R}^{d}, E(p)$ is given by

$$
E(p)=\frac{\left(H_{\epsilon}(p) \Omega(p), \Omega\right)}{(\Omega(p), \Omega)}
$$

Then we get

$$
E(p)=\frac{1}{2 m} \hbar^{2}\left(p^{2}-2 p_{\mu} p_{\nu} \Lambda_{\mu \nu}+\epsilon \sum_{\nu=1}^{d}\left(p_{\mu} \Lambda_{\mu \nu}\right)^{2}\right)-\eta
$$

where

$$
\Lambda_{\mu \nu}=\frac{e}{2 c^{2}}\left(\frac{Q e_{\mu}^{r}}{\sqrt{\omega^{3}}},\left(\mathrm{~W}_{+}^{-1}\right)^{(r, s)} \frac{\hat{\rho} e_{\nu}^{s}}{\sqrt{\omega}}\right)_{0}
$$

Hence it is sufficient to show that $\Lambda_{\mu \nu}=\delta_{\mu \nu} \Lambda$. Let us fix $\lambda_{0}=m c^{2} / \epsilon e^{2}>0$ throughtout this Appendix. Put

$$
H(s)=\frac{d-1}{d} \int_{\mathbb{R}^{d}} \frac{\hat{\rho}(k)^{2}}{s-k^{2}+i 0} d k, s>0 .
$$

Let $\mathbb{R}_{+}=(0, \infty)$, and $\mathbb{R}_{\lambda}=\{x \in \mathbb{R} \mid x \geq \lambda\}$. From the definition of $D_{\epsilon}(s)$ it follows that

$$
D_{\epsilon}(s)=\frac{m}{\lambda_{0}}\left(\lambda_{0}-H(s)\right), s>0 .
$$

From Lemma 5.3 it follows that

$$
\begin{aligned}
& (H .1): \lim _{s \rightarrow \infty} H(s)=0, H(0)=-\frac{d-1}{d}\|\hat{\rho} / \omega\|^{2}<0, \\
& (H .2): \operatorname{Im} H(s)=-\pi \lambda_{0}\left(\frac{d-1}{d} \mathrm{~V}_{d}\right) \hat{\rho}^{2}(\sqrt{s}) s^{\frac{d}{2}-1}<0,(s>0) .
\end{aligned}
$$

Lemma A.1 : For any $\lambda \in \mathbb{R}_{+}$, there exists $M>0$ so that

$$
\operatorname{dist}\left(\operatorname{Ran} H, \mathbb{R}_{\lambda}\right)>M
$$

Proof: From $(H .1)$, for any $0<\epsilon<\lambda$, there exist $s_{1}>0, s_{0}>0$ such that

$$
\begin{aligned}
& |H(s)|<\epsilon, \text { for all } s>s_{1}, \\
& |H(s)-H(0)|<\epsilon, \text { for all } 0<s<s_{0} .
\end{aligned}
$$


By the fact that $H(0)$ is negative number, it follows that

$$
\inf _{0<s<s_{0}, s_{1}<s, x \in \mathbb{R}_{\lambda}}|H(s)-x|>\lambda-\epsilon .
$$

By (H.2), there exists $h>0$ such that $\inf _{s_{0}<s<s_{1}}|\operatorname{Im} H(s)|>h$. Putting $M \equiv \min (\lambda-\epsilon, h)$, it satisfies (A.1).

By Lemma A.1, for $\lambda_{0}$, we can get $h_{\lambda_{0}}>0$ and an open set $\Pi\left(\lambda_{0}\right)$ consisting $\mathbb{R}_{\lambda_{0}}$ such that

$$
\operatorname{dist}\left(\operatorname{Ran} H, \Pi\left(\lambda_{0}\right)\right)>h_{\lambda_{0}} \text {. }
$$

Hence we can define for all $\lambda \in \Pi\left(\lambda_{0}\right)$

$$
T_{\mu \nu}(\lambda) f=\delta_{\mu \nu} f+Q_{\lambda} \omega^{\frac{d}{2}-1} G \omega^{\frac{d}{2}-1} d_{\mu \nu} \hat{\rho} f
$$

where

$$
Q_{\lambda}(s)=\frac{\hat{\rho}}{\lambda-H(s)}
$$

Note that $Q_{\lambda_{0}}=Q_{\epsilon+}$ and $\inf _{s}>0|\lambda-H(s)|>h_{\lambda_{0}}>0$.

Here we regard $T_{\mu \nu}(\lambda)$ as the mapping from $\Pi\left(\lambda_{0}\right)$ to a set of bounded operators:

$$
T_{\mu \nu}(\cdot): \Pi\left(\lambda_{0}\right) \longrightarrow \mathbf{B}\left(M_{\alpha}\right), \alpha= \pm \frac{1}{2}
$$

Lemma A.2 : The operator $T_{\mu \nu}(\lambda)$ can be expanded in a neighborhood of any $\lambda^{\prime} \in \Pi\left(\lambda_{0}\right)$ in operator norm in $\mathbf{B}\left(\mathbf{M}_{\alpha}\right), \alpha= \pm \frac{1}{2}$ : for $\lambda \in \Pi\left(\lambda_{0}\right)$ with $\left|\lambda-\lambda^{\prime}\right|$ sufficiently small,

$$
T_{\mu \nu}(\lambda)=\delta_{\mu \nu} I+\sum_{n=1}^{\infty}(-1)^{n-1}\left(\lambda-\lambda^{\prime}\right)^{n-1} T_{\mu \nu}^{(n-1)}\left(\lambda^{\prime}\right)
$$

where

$$
T_{\mu \nu}^{(n-1)}\left(\lambda^{\prime}\right)=\frac{\hat{\rho}}{\left(\lambda^{\prime}-H\right)^{n}} \omega^{\frac{d}{2}-1} G \omega^{\frac{d}{2}-1} \hat{\rho} d_{\mu \nu},(n \geq 1)
$$

Proof: Noting that

$$
\left\|\frac{1}{\lambda-H}-\sum_{n=1}^{N} \frac{(-1)^{n-1}\left(\lambda-\lambda^{\prime}\right)^{n-1}}{\left(\lambda^{\prime}-H\right)^{n}}\right\|_{\infty} \leq \sum_{n=N+1}^{\infty} \frac{\left|\lambda^{\prime}-\lambda\right|^{n-1}}{h_{\lambda_{0}}^{n}}
$$

one can easily prove Eq.(A.2). 
Let $\mathrm{W}_{ \pm}(\lambda)$ be an operator defined by replacing $T_{\mu \nu}$ by $T_{\mu \nu}(\lambda)$ in $\mathbf{W}_{ \pm}$. Since $T_{\mu \nu}(\lambda)$ is $\mathbf{B}\left(M_{\alpha}\right)$ valued holomorphic function on $\Pi\left(\lambda_{0}\right),\left(\alpha= \pm \frac{1}{2}\right)$, so is $T_{\mu \nu}^{*}(\lambda)$. Hence by the definition of $\mathrm{W}_{ \pm}(\lambda)$, it can be seen $\mathbf{W}_{ \pm}(\lambda)$ is $\mathbf{B}(\mathcal{W})$-valued holomorphic function on $\Pi\left(\lambda_{0}\right)$. We put for $\lambda \in \Pi\left(\lambda_{0}\right)$,

$$
\begin{aligned}
& \mathbf{W}_{+}^{*}(\lambda) \mathbf{W}_{+}(\lambda)-\mathbf{W}_{-}^{*}(\lambda) \mathbf{W}_{-}(\lambda)=\Delta_{1}(\lambda) \\
& \mathbf{W}_{+}(\lambda) \mathbf{W}_{+}^{*}(\lambda)-\bar{W}_{-}(\lambda) \bar{W}_{-}^{*}(\lambda)=\Delta_{2}(\lambda) .
\end{aligned}
$$

By the fact that for all $\lambda^{\prime} \in \mathbb{R}_{+}$,

$$
\begin{aligned}
& \mathbf{W}_{+}^{*}(\lambda) \mathbf{W}_{+}(\lambda)-\mathrm{W}_{-}^{*}(\lambda) \mathrm{W}_{-}(\lambda)=I \\
& \mathrm{~W}_{+}(\lambda) \mathrm{W}_{+}^{*}(\lambda)-\overline{\mathrm{W}}_{-}(\lambda) \overline{\mathrm{W}}_{-}^{*}(\lambda)=I
\end{aligned}
$$

we have that

$$
\lim _{\lambda \rightarrow \lambda^{\prime}} \Delta_{j}(\lambda)=I(j=1,2) \text { in operator norm. }
$$

Hence one can take an open set $\Pi_{\epsilon}\left(\lambda_{0}\right)$ such that

$$
\begin{gathered}
\mathbb{R}_{\lambda_{0}} \subset \Pi_{\epsilon}\left(\lambda_{0}\right) \subset \Pi\left(\lambda_{0}\right), \\
\left\|\Delta_{j}(\lambda)-I\right\|<\epsilon<1(j=1,2), \text { for all } \lambda \in \Pi_{\epsilon}\left(\lambda_{0}\right) .
\end{gathered}
$$

In what follows, let fix the open set $\Pi_{\epsilon}\left(\lambda_{0}\right)$.

Lemma A.3 : For all $\lambda \in \Pi_{\epsilon}\left(\lambda_{0}\right), \mathrm{W}_{+}(\lambda)$ is bijective on $\mathcal{W}$.

Proof: It follows from (A.3) that $\Delta_{j}(\lambda)>1-\epsilon>0, j=1,2$. Hence we have for all $\lambda \in \Pi_{\epsilon}\left(\lambda_{0}\right)$,

$$
\begin{aligned}
& \mathbf{W}_{+}^{*}(\lambda) \mathbf{W}_{+}(\lambda)=\mathbf{W}_{-}^{*}(\lambda) \mathbf{W}_{-}(\lambda)+\Delta_{1}(\lambda)>0 \\
& \mathbf{W}_{+}(\lambda) \mathbf{W}_{+}^{*}(\lambda)=\overline{\mathbf{W}}_{-}(\lambda) \overline{\mathbf{W}}_{-}^{*}(\lambda)+\Delta_{2}(\lambda)>0 .
\end{aligned}
$$

Then we see that $\mathrm{W}_{+}^{*}(\lambda) \mathrm{W}_{+}(\lambda)$ and $\mathrm{W}_{+}(\lambda) \mathrm{W}_{+}^{*}(\lambda)$ are strictly positive self-adjoint operators on $\mathcal{W}$. Thus Lemma A.3 follows.

Lemma A.4 : The operator $\mathrm{W}_{+}(\lambda)^{-1}$ is continous in $\lambda \in \Pi_{\epsilon}\left(\lambda_{0}\right)$ in operator norm. 
Proof: For any $\lambda \in \Pi_{\epsilon}\left(\lambda_{0}\right)$, by the proof of Lemma A.3, we see that

$$
\left\|\mathbf{W}_{+}(\lambda)^{-1}\right\| \leq(1-\epsilon)^{-1}\left\|\mathbf{W}_{+}^{*}(\lambda)\right\|
$$

Since $\left\|\mathbf{W}_{+}^{*}(\lambda)\right\|$ is locally bounded in $\lambda$, for all $h \in \mathbb{C}$ with $|h|$ sufficiently small, there exists $B_{\lambda}<\infty$ such that $\left\|\mathrm{W}_{+}^{*}(\lambda+h)\right\|<B_{\lambda}$. Hence for $h$ stated above,

$$
\left\|\mathrm{W}_{+}(\lambda)^{-1}-\mathrm{W}_{+}(\lambda+h)^{-1}\right\| \leq(1-\epsilon)^{-2} B_{\lambda}^{2}\left\|\mathrm{~W}_{+}(\lambda)-\mathrm{W}_{+}(\lambda+h)\right\|
$$

Thus Lemma A.3 follows.

Lemma A.5 : The operator $\mathrm{W}_{+}(\lambda)^{-1}$ is differentiable in $\lambda \in \Pi_{\epsilon}\left(\lambda_{0}\right)$ in operator norm.

Proof: One can straightforwardly see that

$$
\begin{aligned}
& \lim _{|h| \rightarrow 0}\left\|\frac{\mathbf{W}_{+}(\lambda)^{-1}-\mathbf{W}_{+}(\lambda+h)^{-1}}{h}-\mathbf{W}_{+}(\lambda)^{-1} \mathbf{W}_{+}(\lambda)^{\prime} \mathbf{W}_{+}(\lambda)^{-1}\right\| \\
& \leq \lim _{|h| \rightarrow 0}\left\|\mathbf{W}_{+}(\lambda)^{-1}\right\|\left\{\left\|\mathbf{W}_{+}(\lambda)^{\prime}-\frac{\mathbf{W}_{+}(\lambda+h)-\mathbf{W}_{+}(\lambda)}{h}\right\|\left\|\mathbf{W}_{+}(\lambda)^{-1}\right\|\right. \\
& \left.\quad+\left\|\frac{\mathbf{W}_{+}(\lambda+h)-\mathbf{W}_{+}(\lambda)}{h}\right\|\left\|\mathbf{W}_{+}(\lambda+h)^{-1}-\mathbf{W}_{+}(\lambda)^{-1}\right\|\right\} .
\end{aligned}
$$

By the continuity of $W_{+}(\lambda)^{-1}$ and differentiability of $W_{+}(\lambda)$ in $\lambda$, the r.h.s of (A.4) converges to 0 as $|h| \rightarrow 0$. Thus Lemma A.5 holds.

We have

$$
\left(I-\mathrm{W}_{+}(\lambda)\right)^{(r, s)}=e_{\mu}^{r} \mathrm{Y}(\lambda) e_{\mu}^{s}
$$

where

$$
Y(\lambda)=\hat{\rho}\left(\omega^{\frac{d-3}{2}} G \omega^{\frac{d-1}{2}}+\omega^{\frac{d-1}{2}} G \omega^{\frac{d-3}{2}}\right) \bar{Q}(\lambda)
$$

Lemma A.6 : If $\lambda \in \mathbb{R}_{+}$is sufficientry large, then

$$
\left\|\mathrm{W}_{+}(\lambda)-I\right\|<1
$$

Proof: Let $\sup _{0<s}|H(s)|=M_{H}<\infty$. For $\lambda \in \mathbb{R}_{+}$with $\lambda>M_{H}$, we have for all $f \in L^{2}\left(\mathbb{R}^{d}\right)$,

$$
\left\|e_{\mu}^{\tau} Y e_{\mu}^{s} f\right\| \leq \frac{\|f\|}{\lambda-M_{H}} \times \text { const. }
$$


Then for sufficientry large $\lambda \in \mathbb{R}_{+}$, the r.h.s. of (A.6) is less than 1. Thus (A.5) holds.

We define a complex-valued function on $\Pi_{\epsilon}\left(\lambda_{0}\right)$ by

$$
\Lambda_{\mu \nu}(\cdot)=\frac{1}{2 \epsilon}\left(\frac{Q(\cdot) e_{\mu}^{r}}{\sqrt{\omega^{3}}},\left(\mathrm{~W}_{+}(\cdot)^{-1}\right)^{(r, s)} \frac{\hat{\rho} e_{\nu}^{s}}{\sqrt{\omega}}\right)_{0} .
$$

Then, by Lemma A.6, for sufficiently large $\lambda \in \mathbb{R}_{\lambda_{0}}$, we have that

$$
\Lambda_{\mu \nu}(\lambda)=\sum_{n=1}^{\infty} \frac{1}{2 \epsilon}\left(\frac{Q(\lambda) e_{\mu}^{r}}{\sqrt{\omega^{3}}},\left(\left(I-\mathrm{W}_{+}(\lambda)\right)^{n-1}\right)^{(r, s)} \frac{\hat{\rho} e_{\nu}^{s}}{\sqrt{\omega}}\right)_{0}
$$

Lemma A.7 : If $\lambda \in \mathbb{R}_{\lambda_{0}}$ is sufficientry large, then $\Lambda_{\mu \nu}(\lambda)=\delta_{\mu \nu} \times \Lambda(\lambda)$, where $\Lambda(\lambda)$ is a function on $\Pi_{\epsilon}\left(\lambda_{0}\right)$ independent of $\mu$ and $\nu$.

Proof: Note that for any rotation invariant function $\varrho$,

$$
e_{\mu}^{r} \mathrm{Y}(\lambda) e_{\mu}^{s} e_{\nu}^{s} \varrho=\left(\frac{d-1}{d}\right) e_{\nu}^{r} \mathrm{Y}(\lambda) \varrho
$$

Hence one can easily see that

$$
\begin{aligned}
\left(\left(I-\mathbf{W}_{+}(\lambda)\right)^{n-1}\right)^{(r, s)} \frac{\hat{\rho} e_{\nu}^{s}}{\sqrt{\omega}} & =\sum_{t_{1}, \ldots, t_{n-2}} e_{\mu_{1}}^{r} Y e_{\mu_{1}}^{t_{1}} e_{\mu_{2}}^{t_{1}} Y e_{\mu_{2}}^{t_{2}} \ldots e_{\mu_{n-2}}^{t_{n-2}} Y e_{\mu_{n-2}}^{s} \frac{\hat{\rho} e_{\nu}^{s}}{\sqrt{\omega}} \\
& =\left(\frac{d-1}{d}\right)^{n-1} e_{\nu}^{r} Y^{n-1}(\lambda) \frac{\hat{\rho}}{\sqrt{\omega}}
\end{aligned}
$$

Hence we get

$$
\Lambda_{\mu \nu}(\lambda)=\sum_{n=1}^{\infty} \frac{1}{2 \epsilon}\left(\frac{d-1}{d}\right)^{n}\left(\frac{Q(\lambda)}{\sqrt{\omega^{3}}}, \mathrm{Y}^{n-1}(\lambda) \frac{\hat{\rho}}{\sqrt{\omega}}\right) \delta_{\mu \nu} \equiv \Lambda(\lambda) \delta_{\mu \nu}
$$

Thus we obtain the desired result.

Now we prove Lemma 5.12. By Lemma A.5, the function $\Lambda_{\mu \nu}(\lambda)$ is holomorphic on $\Pi_{\epsilon}\left(\lambda_{0}\right)$, and by the construction of $\Pi_{\epsilon}\left(\lambda_{0}\right)$, the real line $\mathbb{R}_{\lambda_{0}}$ is included in $\Pi_{\epsilon}\left(\lambda_{0}\right)$. Then by Lemma A.7 and theorem of identity in complex analysis, we can conclude that for all $\lambda \in \mathbb{R}_{\lambda_{0}}$

$$
\Lambda_{\mu \nu}(\lambda)=0(\mu \neq \nu), \Lambda_{11}(\lambda)=\ldots=\Lambda_{d d}(\lambda)=\Lambda(\lambda)
$$

Since $\lambda_{0}>0$ is an arbitrarily given real number, it follows that $\Lambda_{\mu \nu}(\lambda)=0(\mu \neq \nu)$ and $\Lambda_{11}(\lambda)=$ $\ldots=\Lambda_{d d}(\lambda)=\Lambda(\lambda)$ for any $\lambda \in \mathbb{B}_{+}$. This completes the proof of Lemma 5.12. 


\section{APPENDIX B}

In this appendix, we construct the Heisenberg field in a general case. We define operators in $\mathcal{F}(\mathcal{H})$, $C_{\mu}(p)$ and $D_{\mu}(p)$, by

$$
\begin{aligned}
& C_{\mu}(p)=\frac{\gamma}{\sqrt{2 E c}}\left(\frac{1}{\sqrt{\epsilon} E} \hbar p_{\mu}+c E \widehat{A}_{\alpha}\left(F_{\alpha \mu}\right)+\widehat{\Pi}_{\alpha}\left(F_{\alpha \mu}\right)\right) \\
& D_{\mu}(p)=\frac{\gamma}{\sqrt{2 E c}}\left(-\frac{1}{\sqrt{\epsilon} E} \hbar p_{\mu}-c E \widehat{A}_{\alpha}\left(F_{\alpha \mu}\right)+\widehat{\Pi}_{\alpha}\left(F_{\alpha \mu}\right)\right) .
\end{aligned}
$$

Lemma B.1 : $C_{\mu}(p)$ and $D_{\nu}(p)$ are essentially self-adjoint on $\mathcal{F}_{0}^{E M}$ and satisfy following commutation relations on $\mathcal{F}_{0}^{E M}$.

$$
\begin{aligned}
& \text { (1) }\left[C_{\mu}(p), D_{\nu}(p)\right]=\delta_{\mu \nu} i \hbar \\
& \text { (2) }\left[C_{\mu}(p), C_{\nu}(p)\right]=\left[D_{\mu}(p), D_{\nu}(p)\right]=0, \\
& \text { (3) }\left[C_{\mu}(p), B^{\sharp(r)}(f, p)\right]=\left[D_{\mu}(p), B^{\sharp(r)}(f, p)\right]=0 .
\end{aligned}
$$

Proof: The proof of the essentially self-adjointness is similar to that of Theorem 5.6. (1) follows from Lemma 5.4 (14). (2) is clear. (3) follws from Lemma 5.4 (11).

Lemma B.2 : The following commutation relations hold on $\mathcal{F}_{0}^{E M} \cap D\left(H^{E M \frac{3}{2}}\right)$ :

$$
\begin{aligned}
& {\left[H_{\epsilon}(p), D_{\mu}(p)\right]=i \hbar c E D_{\mu}(p),} \\
& {\left[H_{\epsilon}(p), C_{\mu}(p)\right]=-i \hbar c E C_{\mu}(p) .}
\end{aligned}
$$

Proof: By Lemma 5.4 (12), one can easily obtain the desired result.

Now we can construct the Heisenberg field in the general case. We define

$$
\begin{aligned}
\tilde{A}_{\mu}(f, t \mid p)= & \frac{1}{\sqrt{2}} \sum_{r=1}^{d-1}\left\{B^{\dagger(r)}\left(\frac{\sqrt{\hbar}}{\sqrt{c \omega}} e^{i c \omega t} e_{\nu}^{r} \bar{T}_{\mu \nu} \hat{f}, p\right)+B^{(r)}\left(\frac{\sqrt{\hbar}}{\sqrt{c \omega}} e^{-i c \omega t} e_{\nu}^{r} T_{\mu \nu} \tilde{\hat{f}}, p\right)\right\} \\
& +\frac{\theta \gamma}{\sqrt{2 E c}}\left(\sqrt{c \omega} F_{\mu \nu}, \frac{\hat{f}}{\sqrt{c \omega}}\right)\left(C_{\nu}(p) e^{t c E}-D_{\nu}(p) e^{-t c E}\right) \\
& -\frac{e}{m+\delta} \hbar p_{\nu}\left(d_{\mu \nu} \frac{\hat{\rho}}{\sqrt{(c \omega)}}, \frac{\hat{f}}{\sqrt{c \omega}}\right), \quad\left(\hat{f} \in M_{-\frac{1}{2}}\right), \\
\tilde{\mathrm{I}}_{\mu}(f, t \mid p)= & \frac{i}{\sqrt{2}} \sum_{r=1}^{d-1}\left\{B^{\dagger(r)}\left(\sqrt{c \omega} \sqrt{\hbar} e^{i c \omega t} e_{\nu}^{r} \bar{T}_{\mu \nu} \hat{f}, p\right)-B^{(r)}\left(\sqrt{c \omega} \sqrt{\hbar} e^{-i c \omega t} e_{\nu}^{r} T_{\mu \nu} \tilde{\hat{f}}, p\right)\right\} \\
& +\frac{\theta \gamma \sqrt{E c}}{\sqrt{2}}\left(\frac{F_{\mu \nu}}{\sqrt{c \omega}}, \sqrt{c \omega} \hat{f}\right)\left(C_{\nu}(p) e^{t c E}+D_{\nu}(p) e^{-t c E}\right), \quad\left(\hat{f} \in M_{\frac{1}{2}}\right) .
\end{aligned}
$$


Theorem B.3 : The operator $\tilde{A}_{\mu}(f, t \mid p),\left(\hat{f} \in M_{-\frac{1}{2}} \cap M_{\frac{1}{2}}\right)$ and $\tilde{\Pi}_{\mu}(f, t \mid p),\left(\hat{f} \in M_{\frac{1}{2}} \cap M_{\frac{3}{2}}\right)$ satisfy the Heisenberg equations Eqs.(4.2) and (4.3), respectively, on $\mathcal{F}_{0}^{E M} \cap D\left(H^{E M \frac{3}{2}}\right)$ with initial condition $\tilde{A}_{\mu}(f, 0 \mid p)=A_{\mu}(f)$ and $\tilde{\Pi}_{\mu}(f, 0 \mid p)=\Pi_{\mu}(f)$, respectively. Furthermore if $f$ is real, then for each $t \in \mathbb{R}, \tilde{A}_{\mu}(f, t \mid p)$ and $\tilde{\Pi}_{\mu}(f, t \mid p)$ are essentially self-adjoint operators on $\mathcal{F}_{0}^{E M}$.

Proof: Similar to that of Lemma 5.6.

Lemma B.4 : The operators $\tilde{A}_{\mu}(f, t \mid p)$ and $\tilde{\Pi}_{\mu}(f, t \mid p)$ satisfy the following equations:

$$
\begin{aligned}
& \text { (1) } \tilde{A}_{\mu}(f, t \mid p)=\exp \left(i \frac{t}{\hbar} H_{\epsilon}(p)\right) A_{\mu}(f) \exp \left(-i \frac{t}{\hbar} H_{\epsilon}(p)\right), \hat{f} \in M_{-\frac{1}{2}}, \\
& \text { (2) } \tilde{\Pi}_{\mu}(f, t \mid p)=\exp \left(i \frac{t}{\hbar} H_{\epsilon}(p)\right) \Pi_{\mu}(f) \exp \left(-i \frac{t}{\hbar} H_{\epsilon}(p)\right), \hat{f} \in M_{\frac{1}{2}} .
\end{aligned}
$$

Proof: Similar to that of Lemma 5.7.

Using Lemma $5.4(5)$ and (6), in the general case we get

$$
\begin{aligned}
& \mathrm{W}_{+}^{*} \mathrm{~W}_{+}-\mathrm{W}_{-}^{*} \mathrm{~W}_{-}=I \\
& \overline{\mathrm{W}}_{+}^{*} \mathrm{~W}_{-}-\overline{\mathrm{W}}_{-}^{*} \mathrm{~W}_{+}=0 \\
& \mathrm{~W}_{+} \mathrm{W}_{+}^{*}-\overline{\mathrm{W}}_{-} \overline{\mathrm{W}}_{-}^{*}=I+\theta \mathrm{Z}_{+} \\
& \mathrm{W}_{-} \mathrm{W}_{+}^{*}-\overline{\mathrm{W}}_{+} \overline{\mathrm{W}}_{-}^{*}=\theta \mathrm{Z}_{-}
\end{aligned}
$$

where

$$
\begin{aligned}
\mathrm{Z}_{ \pm}^{(r, s)} & =\mp \frac{1}{2} \gamma^{2}\left\{\sqrt{\omega} F_{\mu}^{(r)}\left(\frac{F_{\mu}^{(s)}}{\sqrt{\omega}}, \cdot\right) \pm \frac{1}{\sqrt{\omega}} F_{\mu}^{(r)}\left(\sqrt{\omega} F_{\mu}^{(s)}, \cdot\right)\right\} \\
F_{\mu}^{(r)} & =F_{\mu \nu} e_{\nu}^{r}
\end{aligned}
$$

This implies that in the case $m<0$

$$
\Xi(W) \notin \Sigma \text {. }
$$




\section{REFERENCES}

1 A.Arai, "An asymptotic analysis and its application to the nonrelativistic limit of the Pauli-Fierz and a spin-boson model”, J.Math.Phys.31; 2653(1990).

2 H.A.Bethe, "The electromagnetic shift of energy levels", Phys.Rev.72, 339(1947).

3 T.A.Welton, "Some observable effects of the quantum mechanical fluctuations of the electromagnetic field", Phys.Rev.74, 1157(1948).

4 A.Arai, "Noninvertible Bogoliubov transformations and instability of embeded eigenvalues", J.Math.Phys.32, 1838(1991).

5 S.N.M.Ruijsenaars, "On Bogoliubov transformations for systems of relativistic charged particles", J.Math.Phys.18, 517(1977).

6 S.N.M.Ruijsenaars, "Charged particles in external fields. I. Classical theory" J.Math.Phys.18, $720(1977)$.

7 S.N.M.Ruijsenaars, "On Bogoliubov transformations. II. The general case", Ann.Phys.116, 105(1978).

8 A.Arai, "Pertabation of embeded eigenvalues :a general class of exactly soluble models in Fock spaces”, Hokkaido Math.J.19, 1(1990).

9 P.Kristensen, L.Mejlbo and E.Thue.Poulsen, "Tempered distributions in infinitely many dimensions II, displacement operators", Math.scand.14, 129(1964).

10 M.Reed and B.Simon, "Methods of Modern Mathematical Physics IV", Academic Press, New York, 1978.

11 A.Arai, "A note on scattering theory in nonrelativistic quantum electrodynamics", J.Phys.A.Math. Gen.16, 49(1983).

12 A.Arai, "On a model of a harmonic oscillator coupled to a quantized, massless, scalerfield I", J.Math.Phys.22, 2539(1981).

14 A.Arai, "Rigorous theory of spectra and radiation for a model in a quantum electrodynamics", J.Math.Phys. 24, 1896(1983). 